\title{
Entropy stable adaptive moving mesh schemes for 2D and 3D special relativistic hydrodynamics
}

\author{
Junming Duan, Huazhong Tang* \\ Center for Applied Physics and Technology, HEDPS and LMAM, School of Mathematical Sciences, Peking \\ University, Beijing 100871, P.R. China
}

\begin{abstract}
This paper develops entropy stable (ES) adaptive moving mesh schemes for the 2D and 3D special relativistic hydrodynamic (RHD) equations. They are built on the ES finite volume approximation of the RHD equations in curvilinear coordinates, the discrete geometric conservation laws, and the mesh adaptation implemented by iteratively solving the Euler-Lagrange equations of the mesh adaption functional in the computational domain with suitably chosen monitor functions. First, a sufficient condition is proved for the two-point entropy conservative (EC) flux, by mimicking the derivation of the continuous entropy identity in curvilinear coordinates and using the discrete geometric conservation laws given by the conservative metrics method. Based on such sufficient condition, the EC fluxes for the RHD equations in curvilinear coordinates are derived and the second-order accurate semi-discrete EC schemes are developed to satisfy the entropy identity for the given convex entropy pair. Next, the semi-discrete ES schemes satisfying the entropy inequality are proposed by adding a suitable dissipation term to the EC scheme and utilizing linear reconstruction with the minmod limiter in the scaled entropy variables in order to suppress the numerical oscillations of the above EC scheme. Then, the semi-discrete ES schemes are integrated in time by using the second-order strong stability preserving explicit Runge-Kutta schemes. Finally, several numerical results show that our 2D and 3D ES adaptive moving mesh schemes effectively capture the localized structures, such as sharp transitions or discontinuities, and are more efficient than their counterparts on uniform mesh.
\end{abstract}

Keywords: Entropy conservative flux, entropy stable scheme, moving mesh scheme, mesh adaptation, special relativistic hydrodynamics

\footnotetext{
${ }^{*}$ Corresponding author. Fax: +86-10-62751801.

Email addresses: duanjm@pku.edu.cn (Junming Duan), hztang@math.pku.edu.cn (Huazhong Tang)
} 


\section{Introduction}

This paper is concerned with the entropy stable (ES) adaptive moving mesh schemes for the special relativistic hydrodynamic (RHD) equations. In the laboratory frame, the 2D and 3D special RHD equations can be cast in the divergence form

$$
\frac{\partial \boldsymbol{U}}{\partial t}+\sum_{k=1}^{d} \frac{\partial \boldsymbol{F}_{k}(\boldsymbol{U})}{\partial x_{k}}=0, d=2,3,
$$

where $\boldsymbol{U}$ and $\boldsymbol{F}_{k}$ are respectively the conservative vector and the flux vector in the $x_{k}$-direction and defined by

$$
\boldsymbol{U}=\left(\begin{array}{c}
D \\
\boldsymbol{m} \\
E
\end{array}\right), \quad \boldsymbol{F}_{k}=\left(\begin{array}{c}
D v_{k} \\
\boldsymbol{m} v_{k}+p \boldsymbol{e}_{k} \\
m_{k}
\end{array}\right), k=1, \cdots, d,
$$

with the mass density $D=\rho W$, the momentum density $\boldsymbol{m}=\left(m_{1}, \cdots, m_{d}\right)^{\mathrm{T}}=D h W \boldsymbol{v}$, the energy density $E=D h W-p$, the pressure $p$, the fluid velocity $\boldsymbol{v}=\left(v_{1}, \cdots, v_{d}\right)^{\mathrm{T}}$, and the rest-mass density $\rho$. Here $\boldsymbol{e}_{k}$ is the $k$ th column of the unit $d \times d$ matrix, $k=1, \cdots, d, W=1 / \sqrt{1-|\boldsymbol{v}|^{2}}$ is the Lorentz factor and $h=1+e+p / \rho$ is the specific enthalpy with the specific internal energy $e$ and units in which the speed of light is equal to one. The governing equations (1.1)-(1.2) need to be closed by the equation of state (EOS). This paper will only consider the perfect gas with the simple EOS given by

$$
p=(\Gamma-1) \rho e
$$

with the adiabatic index $\Gamma \in(1,2]$. Since there is no explicit expression for the primitive variables $\left(\rho, \boldsymbol{v}^{\mathrm{T}}, p\right)$ and the flux $\boldsymbol{F}_{k}$ in terms of $\boldsymbol{U}$, a nonlinear algebraic equation such as

$$
E+p=D W+\frac{\Gamma}{\Gamma-1} p W^{2}
$$

needs to be (numerically) solved in order to recover the value of the pressure $p$ from the given $\boldsymbol{U}$ and then the rest-mass density $\rho$, the specific enthalpy $h$, and the velocity $\boldsymbol{v}$ by using

$$
\rho=\frac{D}{W}, \quad h=1+\frac{\Gamma p}{(\Gamma-1) \rho}, \quad \boldsymbol{v}=\frac{\boldsymbol{m}}{D h} .
$$

The relativistic description for the fluid dynamics at nearly the speed of light should be considered in investigating the astrophysical phenomena from stellar to galactic scales, e.g. coalescing neutron stars, core collapse supernovae, active galactic nuclei, superluminal jets, the formation of black holes, and gamma-ray bursts etc. The system (1.1)-(1.2) becomes much more complicated than the Euler equations in gas dynamics due to the relativistic effect, so its analytic treatment is very 
challenging. Numerical simulation is a powerful way to help us better understand the physical mechanisms in the RHD. The pioneering numerical work may date back to the finite difference methods with the artificial viscosity technique in the Lagrangian coordinates $[37,38]$ and the Eulerian coordinates [55]. Since the early 1990s, the modern shock-capturing methods were extended to the special or general RHD or relativastic magnetohydronamics (RMHD). They include, but are not limited to, the Roe solver [16], the Harten-Lax-van Leer methods [11, 45], the HartenLax-van Leer Contact methods [33, 40], the essentially non-oscillatory (ENO) and the weighted ENO (WENO) methods [11, 12, 52], the piecewise parabolic methods [34, 42], the adaptive mesh refinement method [72], the Runge-Kutta discontinuous Galerkin (DG) methods with WENO limiter [73], the direct Eulerian generalized Riemann problem schemes [61, 65, 67, 68], the adaptive moving mesh method [24], the two-stage fourth-order accurate time discretizations [69] and so on. The readers are also referred to the early review articles $[20,35,36]$ for more references. Recently, the properties of the admissible state set and the physical-constraints-preserving (PCP) numerical schemes were well studied for the RHD and the special RMHD [33, 60, 62, 63, 64].

For the RHD equations (1.1)-(1.2), it is interesting to design a numerical scheme being consistent with the Clausius inequality, i.e., the entropy inequality. For a general quasi-linear hyperbolic conservation laws, the entropy condition is needed to single out the unique physical relevant solution among all the weak solutions. However, in practice, it is very hard to show that the high-order schemes of the scalar conservation laws and the schemes for the hyperbolic system satisfy the entropy inequality for any convex entropy function. In view of this, many researchers are trying to study the high-order accurate entropy conservative (EC) or ES schemes, which satisfy the entropy identity or inequality for a given entropy pair. The second-order EC schemes were studied in [46, 47], and their higher-order extension was considered in [31]. Unfortunately, the EC schemes may become oscillatory near the discontinuities. To suppress possible numerical oscillation, some additional dissipation term has to be added to obtain the ES schemes. Combining the EC flux with the "sign" property of the ENO reconstruction, the arbitrary high-order ES schemes were constructed by using high-order dissipation terms [19]. The ES schemes based on summation-byparts (SBP) operators were developed for the Navier-Stokes equations [17]. Several ES DG schemes were also studied, such as the semi-discrete DG for scalar conservation laws [30], the space-time DG formulation $[2,26]$ and the DG schemes using suitable quadrature rules for the conservation laws on hexahedron meshes $[8,21]$ and unstructured simplex meshes [10]. As a base of those works, constructing the affordable two-point EC flux is key. Recently, the EC or ES schemes were also extended to the shallow water equations [18], the shallow water magnetohydrodynamics [15, 58], the RHD equations [3, 14], the magnetohydrodynamics [9, 57], the RMHD equations [13, 59], and 
so on.

In view of the fact that the solutions of the RHD equations often exhibit localized structures, e.g. containing sharp transitions or discontinuities in relatively localized regions, the adaptive mesh strategy can improve the efficiency and quality of numerical simulation. Up to now, adaptive moving mesh methods have been successfully applied to many problems in science and engineering, see e.g. $[4,5,24,25,27,28,32,44,49,50,54,56,66,71]$. The readers are also referred to the review papers $[6,51]$ and references therein. This paper aims at developing the ES adaptive moving mesh schemes for the 2D and 3D RHD equations (1.1)-(1.2). Our schemes will be built on the ES finite volume approximation of the RHD equations in curvilinear coordinates, the discrete geometric conservation laws, and the mesh adaptation implemented by iteratively solving the Euler-Lagrange equations of the mesh adaption functional in the computational domain with suitably chosen monitor functions. To do that, we first prove a sufficient condition for the two-point EC fluxes and then derive the EC fluxes in curvilinear coordinates by utilizing the procedure in [13]. The key point is that the geometric conservation laws (GCLs) introduced by the coordinate transformation should be satisfied by the discretization of the metrics. The conservative metric method [53] is adopted to guarantee the GCLs and the suitable dissipation term utilizing linear reconstruction with the minmod limiter in the scaled entropy variables is added to the EC flux to get the second-order accurate ES schemes. The final fully discrete schemes are developed by integrated the semi-discrete ES schemes with the second-order accurate explicit strong-stability preserving (SSP) Runge-Kutta (RK) schemes. Two approximations of the volume conservation law are presented and compared.

The paper is organized as follows. Section 2 introduces the entropy conditions for the RHD equations in Cartesian and curvilinear coordinates. Section 3 presents the EC and ES schemes, including the discretization of the metrics, and construction of the two-point EC flux in curvilinear coordinates. Section 4 gives the adaptive moving mesh strategy. Several 2D and 3D numerical experiments are conducted in Section 5 to validate the efficiency and the ability of our schemes in capturing the sharp transitions or discontinuities. Section 6 concludes the work with final remarks.

\section{Entropy conditions for the RHD}

For the RHD equations (1.1)-(1.2) with the EOS (1.3), there exists an entropy pair $\left(\eta, q_{k}\right)$,

$$
\eta(\boldsymbol{U})=-\frac{\rho W s}{\Gamma-1}, \quad q_{k}(\boldsymbol{U})=\eta v_{k},
$$

where $s=\ln \left(p / \rho^{\Gamma}\right)$ is the thermodynamic entropy, $\eta$ is a convex function of $\boldsymbol{U}$ and $\left(\eta, q_{k}\right)$ satisfies

$$
q_{k}^{\prime}(\boldsymbol{U})=\eta^{\prime}(\boldsymbol{U}) \boldsymbol{F}_{k}^{\prime}(\boldsymbol{U}), \quad k=1, \cdots, d
$$


Here $\eta$ and $q_{k}$ are called the entropy function and entropy flux, respectively. From those, we can also define the entropy variables $\boldsymbol{V}$ by

$$
\boldsymbol{V}:=\eta^{\prime}(\boldsymbol{U})^{\mathrm{T}}=\left(\frac{\Gamma-s}{\Gamma-1}+\frac{\rho}{p}, \frac{\rho W \boldsymbol{v}^{\mathrm{T}}}{p},-\frac{\rho W}{p}\right)^{\mathrm{T}},
$$

and the entropy potential $\phi$ and entropy potential flux $\psi_{k}$ by using the conjugate variables as follows

$$
\phi:=\boldsymbol{V}^{\mathrm{T}} \boldsymbol{U}-\eta=\rho W, \quad \psi_{k}:=\boldsymbol{V}^{\mathrm{T}} \boldsymbol{F}_{k}-q_{k}=\rho W v_{k}
$$

respectively.

For the smooth solutions of (1.1)-(1.2) with the entropy pair $\left(\eta, q_{k}\right)$, multiplying (1.1) by $\boldsymbol{V}^{\mathrm{T}}$ left gives the entropy identity

$$
\boldsymbol{V}^{\mathrm{T}}\left(\frac{\partial \boldsymbol{U}}{\partial t}+\sum_{k=1}^{d} \frac{\partial \boldsymbol{F}_{k}(\boldsymbol{U})}{\partial x_{k}}\right)=\frac{\partial \eta(\boldsymbol{U})}{\partial t}+\sum_{k=1}^{d} \frac{\partial q_{k}(\boldsymbol{U})}{\partial x_{k}}=0 .
$$

For the discontinuous solutions, it is replaced with the entropy inequality

$$
\frac{\partial \eta(\boldsymbol{U})}{\partial t}+\sum_{k=1}^{d} \frac{\partial q_{k}(\boldsymbol{U})}{\partial x_{k}} \leqslant 0
$$

which holds in the sense of distributions.

Next, let us derive the RHD equations in curvilinear coordinates and corresponding entropy condition. Let $\Omega_{p}$ be the domain where the physical problem (1.1)-(1.2) is defined, and $\Omega_{c}$ be the computational domain with coordinates $\boldsymbol{\xi}=\left(\xi_{1}, \cdots, \xi_{d}\right)$ that is artificially chosen for the sake of mesh redistribution or movement. Our adaptive moving meshes for $\Omega_{p}$ can be generated as the images of a reference mesh in $\Omega_{c}$ by a time dependent, differentiable, one-to-one coordinate mapping $\boldsymbol{x}=\boldsymbol{x}(\boldsymbol{\xi}, t)$, which can be expanded as

$$
t=\tau, \quad \boldsymbol{x}=\boldsymbol{x}(\boldsymbol{\xi}, \tau), \quad \boldsymbol{\xi}=\left(\xi_{1}, \cdots, \xi_{d}\right) \in \Omega_{c}
$$

Under this transformation, the detailed transformation of the system (1.1)-(1.2) in the coordinates $(\boldsymbol{\xi}, \tau)$ reads

$$
\frac{\partial(J \boldsymbol{U})}{\partial \tau}+\sum_{k=1}^{d} \frac{\partial}{\partial \xi_{k}}\left(J \frac{\partial \xi_{k}}{\partial t} \boldsymbol{U}\right)+\sum_{k, l=1}^{d} \frac{\partial}{\partial \xi_{k}}\left(J \frac{\partial \xi_{k}}{\partial x_{l}} \boldsymbol{F}_{l}\right)=0,
$$

where $J$ denotes the determinant of the Jacobian matrix and its $3 \mathrm{D}$ version is explicitly given by

$$
J=\operatorname{det}\left(\frac{\partial(t, \boldsymbol{x})}{\partial(\tau, \boldsymbol{\xi})}\right)=\left|\begin{array}{cccc}
1 & 0 & 0 & 0 \\
\frac{\partial x_{1}}{\partial \tau} & \frac{\partial x_{1}}{\partial \xi_{1}} & \frac{\partial x_{1}}{\partial \xi_{2}} & \frac{\partial x_{1}}{\partial \xi_{3}} \\
\frac{\partial x_{2}}{\partial \tau} & \frac{\partial x_{2}}{\partial \xi_{1}} & \frac{\partial x_{2}}{\partial \xi_{2}} & \frac{\partial x_{2}}{\partial \xi_{3}} \\
\frac{\partial x_{3}}{\partial \tau} & \frac{\partial x_{3}}{\partial \xi_{1}} & \frac{\partial x_{3}}{\partial \xi_{2}} & \frac{\partial x_{3}}{\partial \xi_{3}}
\end{array}\right| .
$$


The metric coefficients should satisfy the following geometric conservation laws (GCLs) consisting of the the volume conservation law (VCL) and surface conservation laws (SCLs)

$$
\begin{array}{ll}
\text { VCL: } & \frac{\partial J}{\partial \tau}+\sum_{k=1}^{d} \frac{\partial}{\partial \xi_{k}}\left(J \frac{\partial \xi_{k}}{\partial t}\right)=0, \\
\text { SCLs: } & \sum_{k=1}^{d} \frac{\partial}{\partial \xi_{k}}\left(J \frac{\partial \xi_{k}}{\partial x_{l}}\right)=0, l=1, \cdots, d .
\end{array}
$$

The former indicates that volumetric increment of a moving cell must be equal to the sum of the changes along the surfaces that enclose the volume, while the latter indicates that cell volumes must be closed by its surfaces [70]. Those GCLs mean that free-stream solution is preserved by (2.3), that is to say, if a physical constant state is given as the initial condition, it will remain unchanged. If the free-stream solution cannot be preserved by the numerical schemes on the moving mesh, it may cause some large errors.

Finally, let us derive the entropy identity for the RHD equations (2.3). The three parts of the left-hand side of the product of $\boldsymbol{V}^{\mathrm{T}}$ and (2.3) can be respectively rewritten as follows

$$
\begin{aligned}
& \boldsymbol{V}^{\mathrm{T}} \frac{\partial(J \boldsymbol{U})}{\partial \tau}=\frac{\partial(J \eta)}{\partial \tau}+\left(\boldsymbol{V}^{\mathrm{T}} \boldsymbol{U}-\eta\right) \frac{\partial J}{\partial \tau}, \\
& \boldsymbol{V}^{\mathrm{T}} \sum_{k=1}^{d} \frac{\partial}{\partial \xi_{k}}\left(J \frac{\partial \xi_{k}}{\partial t} \boldsymbol{U}\right)=\sum_{k=1}^{d} \frac{\partial}{\partial \xi_{k}}\left(J \frac{\partial \xi_{k}}{\partial t} \eta\right)+\left(\boldsymbol{V}^{\mathrm{T}} \boldsymbol{U}-\eta\right) \sum_{k=1}^{d} \frac{\partial}{\partial \xi_{k}}\left(J \frac{\partial \xi_{k}}{\partial t}\right), \\
& \boldsymbol{V}^{\mathrm{T}} \sum_{k, l=1}^{d} \frac{\partial}{\partial \xi_{k}}\left(J \frac{\partial \xi_{k}}{\partial x_{l}} \boldsymbol{F}_{l}\right)=\sum_{k, l=1}^{d} \frac{\partial}{\partial \xi_{k}}\left(J \frac{\partial \xi_{k}}{\partial x_{l}} q_{l}\right)+\sum_{l=1}^{d}\left(\boldsymbol{V}^{\mathrm{T}} \boldsymbol{F}_{l}-q_{l}\right) \sum_{k=1}^{d} \frac{\partial}{\partial \xi_{k}}\left(J \frac{\partial \xi_{k}}{\partial x_{l}}\right) .
\end{aligned}
$$

Using the GCLs (2.4) gives

$$
\frac{\partial(J \eta)}{\partial \tau}+\sum_{k=1}^{d} \frac{\partial}{\partial \xi_{k}}\left(J \frac{\partial \xi_{k}}{\partial t} \eta\right)+\sum_{k, l=1}^{d} \frac{\partial}{\partial \xi_{k}}\left(J \frac{\partial \xi_{k}}{\partial x_{l}} q_{l}\right)=0
$$

which is the entropy identity in the coordinates $(\tau, \boldsymbol{\xi})$. Similarly, it will be replaced with corresponding entropy inequality when the solutions $\boldsymbol{U}$ are not smooth.

\section{Numerical schemes}

This section focuses on constructing the 3D moving mesh EC and ES schemes for the RHD equations (2.3) in curvilinear coordinates on the structured mesh. The 2D schemes can be obtained by setting $x_{3}=\xi_{3}$ and removing all the dependence of $\boldsymbol{U}$ on $\xi_{3}$ and $x_{3}, \boldsymbol{F}_{3}$ and the $x_{3}$-component of $\boldsymbol{U}$ and $\boldsymbol{F}_{k}, k=1,2$. In view of $t=\tau$, the symbol $\tau$ will be replaced with $t$ hereafter. 


\subsection{EC scheme}

Assume that the computational domain $\Omega_{c}$ is rectangular, e.g. $[0,1] \times[0,1] \times[0,1]$, and divided into a fixed orthogonal mesh $\left\{\left(\xi_{1, i_{1}+\frac{1}{2}}, \xi_{2, i_{2}+\frac{1}{2}}, \xi_{3, i_{3}+\frac{1}{2}}\right): 0=\xi_{k, \frac{1}{2}}<\xi_{k, 1+\frac{1}{2}}<\cdots<\right.$ $\left.\xi_{k, i_{k}+\frac{1}{2}}<\cdots<\xi_{k, N_{k}+\frac{1}{2}}=1, k=1,2,3\right\}$ with the constant step-size $\Delta \xi_{k}=\xi_{k, i_{k}+\frac{1}{2}}-\xi_{k, i_{k}-\frac{1}{2}}$. For the sake of brevity, the index $\boldsymbol{i}=\left(i_{1}, i_{2}, i_{3}\right)$ is used to denote the cell $\left[\xi_{1, i_{1}-\frac{1}{2}}, \xi_{1, i_{1}+\frac{1}{2}}\right] \times$ $\left[\xi_{2, i_{2}-\frac{1}{2}}, \xi_{2, i_{2}+\frac{1}{2}}\right] \times\left[\xi_{3, i_{3}-\frac{1}{2}}, \xi_{3, i_{3}+\frac{1}{2}}\right]$ and $\hat{\boldsymbol{i}}_{1, \pm}, \cdots, \hat{\boldsymbol{i}}_{3, \pm}$ denote the middle points of the cell interfaces, i.e. $\left(\xi_{1, i_{1} \pm \frac{1}{2}}, \xi_{2, i_{2}}, \xi_{3, i_{3}}\right),\left(\xi_{1, i_{1}}, \xi_{2, i_{2} \pm \frac{1}{2}}, \xi_{3, i_{3}}\right),\left(\xi_{1, i_{1}}, \xi_{2, i_{2}}, \xi_{3, i_{3} \pm \frac{1}{2}}\right)$, respectively, where $\xi_{k, i_{k}}=$ $\left(\xi_{k, i_{k}+\frac{1}{2}}+\xi_{k, i_{k}-\frac{1}{2}}\right) / 2, k=1,2,3$.

For the cell $\boldsymbol{i}$, the RHD system (2.3) and the first equation of (2.4) can be approximated as the following semi-discrete conservative finite volume scheme

$$
\begin{gathered}
\frac{\mathrm{d}}{\mathrm{d} t}(J \boldsymbol{U})_{\boldsymbol{i}}=-\sum_{k=1}^{3} \frac{1}{\Delta \xi_{k}} \delta_{k}\left[\widehat{\boldsymbol{F}}_{k}\right]_{\boldsymbol{i}}, \\
\frac{\mathrm{d}}{\mathrm{d} t} J_{\boldsymbol{i}}=-\sum_{k=1}^{3} \frac{1}{\Delta \xi_{k}} \delta_{k}\left[J \frac{\partial \xi_{k}}{\partial t}\right]_{\boldsymbol{i}},
\end{gathered}
$$

where $\delta_{k}[\cdot]$ is the second-order central difference operator in the $i_{k}$-direction, e.g. $\delta_{k}[a]_{i}=a_{\hat{\boldsymbol{i}}_{k,+}}-$ $a_{\hat{\boldsymbol{i}}_{k,-}}, J_{\boldsymbol{i}}(t)$ and $(J \boldsymbol{U})_{\boldsymbol{i}}(t)$ approximate the cell average values of $J(t, \boldsymbol{\xi})$ and $(J \boldsymbol{U})(t, \boldsymbol{\xi})$ over the cell $\boldsymbol{i}$, respectively, and $\widehat{\boldsymbol{F}}_{k}(t)$ is the numerical flux approximating the flux $\left(J \frac{\partial \xi_{k}}{\partial t} \boldsymbol{U}+\sum_{l=1}^{3} J \frac{\partial \xi_{k}}{\partial x_{l}} \boldsymbol{F}_{l}\right)(t, \boldsymbol{\xi})$, $k=1,2,3$. The metrics $\left(J \frac{\partial \xi_{k}}{\partial t}\right)_{\hat{\boldsymbol{i}}_{k, \pm}}$ and $\left(J \frac{\partial \xi_{k}}{\partial x_{l}}\right)_{\hat{\boldsymbol{i}}_{k, \pm}}$ in (3.1)-(3.2) are calculated by (3.7)-(3.8), see Section 3.2, with which the SCLs in the second equation of (2.4) are satisfied at the discrete level, i.e.

$$
\sum_{k=1}^{3} \frac{1}{\Delta \xi_{k}} \delta_{k}\left[J \frac{\partial \xi_{k}}{\partial x_{l}}\right]_{i}=0, l=1,2,3
$$

Definition 3.1 (EC scheme). The semi-discrete scheme (3.1)-(3.2) is EC and corresponding numerical flux $\widehat{\boldsymbol{F}}_{k}$ is called the EC flux, if its solution satisfies a semi-discrete entropy identity

$$
\frac{\mathrm{d}}{\mathrm{d} t} J_{\boldsymbol{i}} \eta\left(\boldsymbol{U}_{\boldsymbol{i}}(t)\right)+\sum_{k=1}^{3} \frac{1}{\Delta \xi_{k}} \delta_{k}\left[\widetilde{q}_{k}(t)\right]_{\boldsymbol{i}}=0
$$

for some numerical entropy fluxes $\widetilde{q}_{k}$ consistent with the continuous entropy flux $J \frac{\partial \xi_{k}}{\partial t} \eta+\sum_{l=1}^{3} J \frac{\partial \xi_{k}}{\partial x_{l}} q_{l}$.

The following lemma gives a sufficient condition for the semi-discrete scheme (3.1)-(3.2) to be EC.

Lemma 3.1. Assume that symmetric two-point flux $\widetilde{\boldsymbol{F}}_{k, \hat{\boldsymbol{i}}_{k, \pm}}$ is consistent with $J \frac{\partial \xi_{k}}{\partial t} U+\sum_{l=1}^{3} J \frac{\partial \xi_{k}}{\partial x_{l}} \boldsymbol{F}_{l}$, and satisfies

$$
\llbracket \boldsymbol{V} \rrbracket_{\hat{\boldsymbol{i}}_{k, \pm}}^{\mathrm{T}} \cdot \widetilde{\boldsymbol{F}}_{k, \hat{\boldsymbol{i}}_{k, \pm}}=\left(J \frac{\partial \xi_{k}}{\partial t}\right)_{\hat{\boldsymbol{i}}_{k, \pm}} \llbracket \phi \rrbracket_{\hat{\boldsymbol{i}}_{k, \pm}}+\sum_{l=1}^{3}\left(J \frac{\partial \xi_{k}}{\partial x_{l}}\right)_{\hat{\boldsymbol{i}}_{k, \pm}} \llbracket \psi_{l} \rrbracket_{\hat{\boldsymbol{i}}_{k, \pm}}
$$


where $\phi$ and $\psi_{l}$ are defined in (2.1), then the semi-discrete scheme (3.1)-(3.2) with $\widehat{\boldsymbol{F}}_{k}(t)=\widetilde{\boldsymbol{F}}_{k, \hat{\boldsymbol{i}}_{k, \pm}}$ is EC with the numerical entropy fluxes

$$
\widetilde{q}_{k, \hat{\boldsymbol{i}}_{k, \pm}}=\left\{\{\boldsymbol{V}\}_{\hat{\boldsymbol{i}}_{k, \pm}^{\mathrm{T}}}^{\mathrm{T}} \widetilde{\boldsymbol{F}}_{k, \hat{\boldsymbol{i}}_{k, \pm}}-\left(J \frac{\partial \xi_{k}}{\partial t}\right)_{\hat{\boldsymbol{i}}_{k, \pm}}\left\{\{\phi\}_{\hat{\boldsymbol{i}}_{k, \pm}}-\sum_{l=1}^{3}\left(J \frac{\partial \xi_{k}}{\partial x_{l}}\right)_{\hat{\boldsymbol{i}}_{k, \pm}}\left\{\left\{\psi_{l}\right\}_{\hat{\boldsymbol{i}}_{k, \pm}},\right.\right.\right.
$$

where $\llbracket a \rrbracket_{\hat{\boldsymbol{i}}_{k, \pm}}$ and $\left\{\{a\}_{\hat{\boldsymbol{i}}_{k, \pm}}\right.$ denote the jumps and the arithmetic means of $a$ in the $i_{k}$-direction, respectively, e.g.

$$
\begin{aligned}
& \llbracket a \rrbracket_{\hat{\boldsymbol{i}}_{1,+}}=a_{i_{1}+1, i_{2}, i_{3}}-a_{i_{1}, i_{2}, i_{3}}, \llbracket a \rrbracket_{\hat{\boldsymbol{i}}_{2,-}}=a_{i_{1}, i_{2}, i_{3}}-a_{i_{1}, i_{2}-1, i_{3}}, \\
& \left\{\{a\}_{\hat{\boldsymbol{i}}_{1,+}}=\left(a_{i_{1}+1, i_{2}, i_{3}}+a_{i_{1}, i_{2}, i_{3}}\right) / 2, \quad\{a\}_{\hat{\boldsymbol{i}}_{2,-}}=\left(a_{i_{1}, i_{2}, i_{3}}+a_{i_{1}, i_{2}-1, i_{3}}\right) / 2 .\right.
\end{aligned}
$$

Proof. Multiplying (3.1) by $\boldsymbol{V}_{\boldsymbol{i}}^{\mathrm{T}}$ left and using (3.2) gives

$$
\frac{\mathrm{d}}{\mathrm{d} t}\left(J_{\boldsymbol{i}} \eta_{\boldsymbol{i}}\right)=-\sum_{k=1}^{3} \frac{1}{\Delta \xi_{k}}\left\{\boldsymbol{V}_{\boldsymbol{i}}^{\mathrm{T}} \delta_{k}\left[\widetilde{\boldsymbol{F}}_{k}\right]_{\boldsymbol{i}}-\phi_{\boldsymbol{i}} \delta_{k}\left[J \frac{\partial \xi_{k}}{\partial t}\right]_{\boldsymbol{i}}\right\} .
$$

Utilizing the discrete SCLs (3.3) gives

$$
\frac{\mathrm{d}}{\mathrm{d} t}\left(J_{\boldsymbol{i}} \eta_{\boldsymbol{i}}\right)=-\sum_{k=1}^{3} \frac{1}{\Delta \xi_{k}}\left\{\boldsymbol{V}_{\boldsymbol{i}}^{\mathrm{T}} \delta_{k}\left[\widetilde{\boldsymbol{F}}_{k}\right]_{\boldsymbol{i}}-\phi_{\boldsymbol{i}} \delta_{k}\left[J \frac{\partial \xi_{k}}{\partial t}\right]_{\boldsymbol{i}}-\sum_{l=1}^{3} \psi_{l, i} \delta_{k}\left[J \frac{\partial \xi_{k}}{\partial x_{l}}\right]_{\boldsymbol{i}}\right\} .
$$

The term in braces at the right end of the above equation can be further rearranged as follows

$$
\begin{aligned}
& \boldsymbol{V}_{\boldsymbol{i}}^{\mathrm{T}} \delta_{k}\left[\widetilde{\boldsymbol{F}}_{k}\right]_{\boldsymbol{i}}-\phi_{\boldsymbol{i}} \delta_{k}\left[J \frac{\partial \xi_{k}}{\partial t}\right]_{\boldsymbol{i}}-\sum_{l=1}^{3} \psi_{l, i} \delta_{k}\left[J \frac{\partial \xi_{k}}{\partial x_{l}}\right]_{\boldsymbol{i}} \\
= & \left(\{\boldsymbol{V}\}_{\hat{\boldsymbol{i}}_{k,+}}-\frac{1}{2} \llbracket \boldsymbol{V} \rrbracket_{\hat{\boldsymbol{i}}_{k,+}}\right)^{\mathrm{T}} \widetilde{\boldsymbol{F}}_{k, \hat{\boldsymbol{i}}_{k,+}}-\left(\left\{[\boldsymbol{V}\}_{\hat{\boldsymbol{i}}_{k,-}}+\frac{1}{2} \llbracket \boldsymbol{V} \rrbracket_{\hat{\boldsymbol{i}}_{k,-}}\right)^{\mathrm{T}} \widetilde{\boldsymbol{F}}_{k, \hat{\boldsymbol{i}}_{k,-}}\right. \\
& -\left(\left\{\{\phi\}_{\hat{\boldsymbol{i}}_{k,+}}-\frac{1}{2} \llbracket \phi \rrbracket_{\hat{\boldsymbol{i}}_{k,+}}\right)\left(J \frac{\partial \xi_{k}}{\partial t}\right)_{\hat{\boldsymbol{i}}_{k,+}}+\left(\{\phi\}_{\hat{\boldsymbol{i}}_{k,-}}+\frac{1}{2} \llbracket \phi \rrbracket_{\hat{\boldsymbol{i}}_{k,-}}\right)\left(J \frac{\partial \xi_{k}}{\partial t}\right)_{\hat{\boldsymbol{i}}_{k,-}}\right. \\
& -\sum_{l=1}^{3}\left(\left\{\left\{\psi_{l}\right\}_{\hat{i}_{k,+}}-\frac{1}{2} \llbracket \psi_{l} \rrbracket_{\hat{\boldsymbol{i}}_{k,+}}\right)\left(J \frac{\partial \xi_{k}}{\partial x_{l}}\right)_{\hat{\boldsymbol{i}}_{k,+}}+\sum_{l=1}^{3}\left(\left\{\left\{\psi_{1}\right\}_{\hat{\boldsymbol{i}}_{k,-}}+\frac{1}{2} \llbracket \psi_{1} \rrbracket_{\hat{\boldsymbol{i}}_{k,-}}\right)\left(J \frac{\partial \xi_{k}}{\partial x_{l}}\right)_{\hat{\boldsymbol{i}}_{k,-}}\right.\right. \\
= & \left(\{\boldsymbol{V}\}_{\hat{\boldsymbol{i}}_{k,+}^{\mathrm{T}}}^{\mathrm{T}} \widetilde{\boldsymbol{F}}_{k, \hat{\boldsymbol{i}}_{k,+}}-\left(J \frac{\partial \xi_{k}}{\partial t}\right)_{\hat{\boldsymbol{i}}_{k,+}}\left\{\{\phi\}_{\hat{\boldsymbol{i}}_{k,+}}-\sum_{l=1}^{3}\left(J \frac{\partial \xi_{k}}{\partial x_{l}}\right)_{\hat{\boldsymbol{i}}_{k,+}}\left\{\left\{\psi_{l}\right\}_{\hat{\boldsymbol{i}}_{k,+}}\right)\right.\right. \\
= & \left(\{\boldsymbol{V}\}_{\hat{\boldsymbol{i}}_{k,-}}^{\mathrm{T}} \widetilde{\boldsymbol{F}}_{k, \hat{\boldsymbol{i}}_{k,-}}-\left(J \frac{\partial \xi_{k}}{\partial t}\right)_{\hat{\boldsymbol{i}}_{k,-}}\left\{\{\phi\}_{\hat{\boldsymbol{i}}_{k,-}}-\sum_{l=1}^{3}\left(J \frac{\partial \xi_{k}}{\partial x_{l}}\right)_{\hat{\boldsymbol{i}}_{k,-}}\left\{\left\{\psi_{l}\right\}_{\hat{\boldsymbol{i}}_{k,-}}\right)\right.\right. \\
& -\widetilde{q}_{k, \hat{\boldsymbol{i}}_{k,-}},
\end{aligned}
$$

where $a_{\boldsymbol{i}}=\left\{\{a\}_{\hat{\boldsymbol{i}}_{k,+}}-\frac{1}{2} \llbracket a \rrbracket \rrbracket_{\hat{\boldsymbol{i}}_{k,+}}\right.$ and $a_{\boldsymbol{i}}=\left\{\{a\}_{\hat{\boldsymbol{i}}_{k,-}}+\frac{1}{2} \llbracket a \rrbracket_{\hat{\boldsymbol{i}}_{k,-}}\right.$ have been used in the first equality, and the condition (3.4) has been used in the second equality. Moreover, it is easy to check the 
consistency of the numerical entropy fluxes $\widetilde{q}_{k, \hat{\boldsymbol{i}}_{k, \pm}}$ with $J \frac{\partial \xi_{k}}{\partial t} \eta+\sum_{l=1}^{3} J \frac{\partial \xi_{k}}{\partial x_{l}} q_{l}$. Thus the scheme (3.1) with $\widehat{\boldsymbol{F}}_{k, \hat{\boldsymbol{i}}_{k, \pm}}=\widetilde{\boldsymbol{F}}_{k, \hat{\boldsymbol{i}}_{k, \pm}}$ is EC in the sense of

$$
\frac{\mathrm{d}}{\mathrm{d} t} J_{\boldsymbol{i}} \eta\left(\boldsymbol{U}_{\boldsymbol{i}}(t)\right)+\sum_{k=1}^{3} \frac{1}{\Delta \xi_{k}} \delta_{k}\left[\widetilde{q}_{k}(t)\right]_{\boldsymbol{i}}=0 .
$$

Remark 3.1. The sufficient condition (3.4) is different from that in [14], due to the metrics introduced by the coordinate transformation.

\subsection{Discrete GCLs}

For the transformation (2.2), we have the following identities

$$
J \frac{\partial \xi_{k}}{\partial t}=-\sum_{l=1}^{3} \frac{\partial x_{l}}{\partial t}\left(J \frac{\partial \xi_{k}}{\partial x_{l}}\right), k=1,2,3
$$

and

$$
\begin{aligned}
J \frac{\partial \xi_{1}}{\partial x_{1}} & =\frac{\partial x_{2}}{\partial \xi_{2}} \frac{\partial x_{3}}{\partial \xi_{3}}-\frac{\partial x_{2}}{\partial \xi_{3}} \frac{\partial x_{3}}{\partial \xi_{2}}, J \frac{\partial \xi_{1}}{\partial x_{2}}=\frac{\partial x_{3}}{\partial \xi_{2}} \frac{\partial x_{1}}{\partial \xi_{3}}-\frac{\partial x_{3}}{\partial \xi_{3}} \frac{\partial x_{1}}{\partial \xi_{2}}, J \frac{\partial \xi_{1}}{\partial x_{3}}=\frac{\partial x_{1}}{\partial \xi_{2}} \frac{\partial x_{2}}{\partial \xi_{3}}-\frac{\partial x_{1}}{\partial \xi_{3}} \frac{\partial x_{2}}{\partial \xi_{2}} \\
J \frac{\partial \xi_{2}}{\partial x_{1}} & =\frac{\partial x_{2}}{\partial \xi_{3}} \frac{\partial x_{3}}{\partial \xi_{1}}-\frac{\partial x_{2}}{\partial \xi_{1}} \frac{\partial x_{3}}{\partial \xi_{3}}, J \frac{\partial \xi_{2}}{\partial x_{2}}=\frac{\partial x_{3}}{\partial \xi_{3}} \frac{\partial x_{1}}{\partial \xi_{1}}-\frac{\partial x_{3}}{\partial \xi_{1}} \frac{\partial x_{1}}{\partial \xi_{3}}, J \frac{\partial \xi_{2}}{\partial x_{3}}=\frac{\partial x_{1}}{\partial \xi_{3}} \frac{\partial x_{2}}{\partial \xi_{1}}-\frac{\partial x_{1}}{\partial \xi_{1}} \frac{\partial x_{2}}{\partial \xi_{3}} \\
J \frac{\partial \xi_{3}}{\partial x_{1}} & =\frac{\partial x_{2}}{\partial \xi_{1}} \frac{\partial x_{3}}{\partial \xi_{2}}-\frac{\partial x_{2}}{\partial \xi_{2}} \frac{\partial x_{3}}{\partial \xi_{1}}, J \frac{\partial \xi_{3}}{\partial x_{2}}=\frac{\partial x_{3}}{\partial \xi_{1}} \frac{\partial x_{1}}{\partial \xi_{2}}-\frac{\partial x_{3}}{\partial \xi_{2}} \frac{\partial x_{1}}{\partial \xi_{1}}, J \frac{\partial \xi_{3}}{\partial x_{3}}=\frac{\partial x_{1}}{\partial \xi_{1}} \frac{\partial x_{2}}{\partial \xi_{2}}-\frac{\partial x_{1}}{\partial \xi_{2}} \frac{\partial x_{2}}{\partial \xi_{1}}
\end{aligned}
$$

The last nine identities can be reformulated into the divergence form

$$
\begin{aligned}
& J \frac{\partial \xi_{1}}{\partial x_{1}}=\frac{\partial}{\partial \xi_{3}}\left(\frac{\partial x_{2}}{\partial \xi_{2}} x_{3}\right)-\frac{\partial}{\partial \xi_{2}}\left(\frac{\partial x_{2}}{\partial \xi_{3}} x_{3}\right), J \frac{\partial \xi_{1}}{\partial x_{2}}=\frac{\partial}{\partial \xi_{3}}\left(\frac{\partial x_{3}}{\partial \xi_{2}} x_{1}\right)-\frac{\partial}{\partial \xi_{2}}\left(\frac{\partial x_{3}}{\partial \xi_{3}} x_{1}\right), \\
& J \frac{\partial \xi_{1}}{\partial x_{3}}=\frac{\partial}{\partial \xi_{3}}\left(\frac{\partial x_{1}}{\partial \xi_{2}} x_{2}\right)-\frac{\partial}{\partial \xi_{2}}\left(\frac{\partial x_{1}}{\partial \xi_{3}} x_{2}\right), \\
& J \frac{\partial \xi_{2}}{\partial x_{1}}=\frac{\partial}{\partial \xi_{1}}\left(\frac{\partial x_{2}}{\partial \xi_{3}} x_{3}\right)-\frac{\partial}{\partial \xi_{3}}\left(\frac{\partial x_{2}}{\partial \xi_{1}} x_{3}\right), J \frac{\partial \xi_{2}}{\partial x_{2}}=\frac{\partial}{\partial \xi_{1}}\left(\frac{\partial x_{3}}{\partial \xi_{3}} x_{1}\right)-\frac{\partial}{\partial \xi_{3}}\left(\frac{\partial x_{3}}{\partial \xi_{1}} x_{1}\right), \\
& J \frac{\partial \xi_{2}}{\partial x_{3}}=\frac{\partial}{\partial \xi_{1}}\left(\frac{\partial x_{1}}{\partial \xi_{3}} x_{2}\right)-\frac{\partial}{\partial \xi_{3}}\left(\frac{\partial x_{1}}{\partial \xi_{1}} x_{2}\right), \\
& J \frac{\partial \xi_{3}}{\partial x_{1}}=\frac{\partial}{\partial \xi_{2}}\left(\frac{\partial x_{2}}{\partial \xi_{1}} x_{3}\right)-\frac{\partial}{\partial \xi_{1}}\left(\frac{\partial x_{2}}{\partial \xi_{2}} x_{3}\right), J \frac{\partial \xi_{3}}{\partial x_{2}}=\frac{\partial}{\partial \xi_{2}}\left(\frac{\partial x_{3}}{\partial \xi_{1}} x_{1}\right)-\frac{\partial}{\partial \xi_{1}}\left(\frac{\partial x_{3}}{\partial \xi_{2}} x_{1}\right), \\
& J \frac{\partial \xi_{3}}{\partial x_{3}}=\frac{\partial}{\partial \xi_{2}}\left(\frac{\partial x_{1}}{\partial \xi_{1}} x_{2}\right)-\frac{\partial}{\partial \xi_{1}}\left(\frac{\partial x_{1}}{\partial \xi_{2}} x_{2}\right),
\end{aligned}
$$

which are useful to compute the discrete metrics and to get the discrete SCLs approximating conservatively (2.4) by the so-called conservative metrics method [53]. 
To establish the discrete SCLs (3.3), using the same discretizations for the first-order spatial derivatives in (3.6) as those in (3.1)-(3.2) gives

$$
\begin{aligned}
& \left(J \frac{\partial \xi_{1}}{\partial x_{1}}\right)_{\hat{\boldsymbol{i}}_{1,+}}=\frac{1}{\Delta \xi_{2} \Delta \xi_{3}}\left(\delta_{3}\left[\delta_{2}\left[x_{2}\right] \overline{\overline{x_{3}}}\right]-\delta_{2}\left[\delta_{3}\left[x_{2}\right] \overline{\overline{x_{3}}}\right]\right), \\
& \left(J \frac{\partial \xi_{1}}{\partial x_{2}}\right)_{\hat{\boldsymbol{i}}_{1,+}}=\frac{1}{\Delta \xi_{2} \Delta \xi_{3}}\left(\delta_{3}\left[\delta_{2}\left[x_{3}\right] \overline{\overline{x_{1}}}\right]-\delta_{2}\left[\delta_{3}\left[x_{3}\right] \overline{\overline{x_{1}}}\right]\right) \\
& \left(J \frac{\partial \xi_{1}}{\partial x_{3}}\right)_{\hat{\boldsymbol{i}}_{1,+}}=\frac{1}{\Delta \xi_{2} \Delta \xi_{3}}\left(\delta_{3}\left[\delta_{2}\left[x_{1}\right] \overline{\overline{x_{2}}}\right]-\delta_{2}\left[\delta_{3}\left[x_{1}\right] \overline{\overline{x_{2}}}\right]\right), \\
& \left(J \frac{\partial \xi_{2}}{\partial x_{1}}\right)_{\hat{\boldsymbol{i}}_{2,+}}=\frac{1}{\Delta \xi_{3} \Delta \xi_{1}}\left(\delta_{1}\left[\delta_{3}\left[x_{2}\right] \overline{\overline{x_{3}}}\right]-\delta_{3}\left[\delta_{1}\left[x_{2}\right] \overline{x_{3}}\right]\right) \\
& \left(J \frac{\partial \xi_{2}}{\partial x_{2}}\right)_{\hat{\boldsymbol{i}}_{2,+}}=\frac{1}{\Delta \xi_{3} \Delta \xi_{1}}\left(\delta_{1}\left[\delta_{3}\left[x_{3}\right] \overline{\overline{x_{1}}}\right]-\delta_{3}\left[\delta_{1}\left[x_{3}\right] \overline{x_{1}}\right]\right) \\
& \left(J \frac{\partial \xi_{2}}{\partial x_{3}}\right)_{\hat{\boldsymbol{i}}_{2,+}}=\frac{1}{\Delta \xi_{3} \Delta \xi_{1}}\left(\delta_{1}\left[\delta_{3}\left[x_{1}\right] \overline{\overline{x_{2}}}\right]-\delta_{3}\left[\delta_{1}\left[x_{1}\right] \overline{x_{2}}\right]\right) \\
& \left(J \frac{\partial \xi_{3}}{\partial x_{1}}\right)_{\hat{\boldsymbol{i}}_{3,+}}=\frac{1}{\Delta \xi_{1} \Delta \xi_{2}}\left(\delta_{2}\left[\delta_{1}\left[x_{2}\right] \overline{x_{3}}\right]-\delta_{1}\left[\delta_{2}\left[x_{2}\right] \overline{\overline{x_{3}}}\right]\right) \\
& \left(J \frac{\partial \xi_{3}}{\partial x_{2}}\right)_{\hat{\boldsymbol{i}}_{3,+}}=\frac{1}{\Delta \xi_{1} \Delta \xi_{2}}\left(\delta_{2}\left[\delta_{1}\left[x_{3}\right] \overline{x_{1}}\right]-\delta_{1}\left[\delta_{2}\left[x_{3}\right] \overline{\overline{x_{1}}}\right]\right) \\
& \left(J \frac{\partial \xi_{3}}{\partial x_{3}}\right)_{\hat{\boldsymbol{i}}_{3,+}}=\frac{1}{\Delta \xi_{1} \Delta \xi_{2}}\left(\delta_{1}\left[\delta_{1}\left[x_{1}\right] \overline{x_{2}}\right]-\delta_{1}\left[\delta_{2}\left[x_{1}\right] \overline{\overline{x_{2}}}\right]\right)
\end{aligned}
$$

where $\bar{a}, \overline{\bar{a}}, \overline{\bar{a}}$ denote the averages in the $i_{1}, i_{2}, i_{3}$-directions, respectively. To be more specific, the right hand-side (RHS) of the first equation in (3.7) can be expanded as follows

$$
\begin{aligned}
\frac{1}{2 \Delta \xi_{2} \Delta \xi_{3}}\{ & {\left[\left(x_{2}\right)_{i_{1}+\frac{1}{2}, i_{2}+\frac{1}{2}, i_{3}+\frac{1}{2}}-\left(x_{2}\right)_{i_{1}+\frac{1}{2}, i_{2}-\frac{1}{2}, i_{3}+\frac{1}{2}}\right]\left[\left(x_{3}\right)_{i_{1}+\frac{1}{2}, i_{2}+\frac{1}{2}, i_{3}+\frac{1}{2}}+\left(x_{3}\right)_{i_{1}+\frac{1}{2}, i_{2}-\frac{1}{2}, i_{3}+\frac{1}{2}}\right] } \\
- & {\left[\left(x_{2}\right)_{i_{1}+\frac{1}{2}, i_{2}+\frac{1}{2}, i_{3}-\frac{1}{2}}-\left(x_{2}\right)_{i_{1}+\frac{1}{2}, i_{2}-\frac{1}{2}, i_{3}-\frac{1}{2}}\right]\left[\left(x_{3}\right)_{i_{1}+\frac{1}{2}, i_{2}+\frac{1}{2}, i_{3}-\frac{1}{2}}+\left(x_{3}\right)_{i_{1}+\frac{1}{2}, i_{2}-\frac{1}{2}, i_{3}-\frac{1}{2}}\right] } \\
& -\left[\left(x_{2}\right)_{i_{1}+\frac{1}{2}, i_{2}+\frac{1}{2}, i_{3}+\frac{1}{2}}-\left(x_{2}\right)_{\left.i_{1}+\frac{1}{2}, i_{2}+\frac{1}{2}, i_{3}-\frac{1}{2}\right]}\right]\left[\left(x_{3}\right)_{i_{1}+\frac{1}{2}, i_{2}+\frac{1}{2}, i_{3}+\frac{1}{2}}+\left(x_{3}\right)_{i_{1}+\frac{1}{2}, i_{2}+\frac{1}{2}, i_{3}-\frac{1}{2}}\right] \\
& \left.+\left[\left(x_{2}\right)_{i_{1}+\frac{1}{2}, i_{2}-\frac{1}{2}, i_{3}+\frac{1}{2}}-\left(x_{2}\right)_{i_{1}+\frac{1}{2}, i_{2}-\frac{1}{2}, i_{3}-\frac{1}{2}}\right]\left[\left(x_{3}\right)_{i_{1}+\frac{1}{2}, i_{2}-\frac{1}{2}, i_{3}+\frac{1}{2}}+\left(x_{3}\right)_{i_{1}+\frac{1}{2}, i_{2}-\frac{1}{2}, i_{3}-\frac{1}{2}}\right]\right\} .
\end{aligned}
$$

Based on the above discretizations, it can be verified that the SCLs (3.3) are satisfied. For example,

$$
\begin{aligned}
\sum_{k=1}^{3} \frac{1}{\Delta \xi_{k}} \delta_{k}\left[J \frac{\partial \xi_{k}}{\partial x_{1}}\right]_{i}=\frac{1}{2 \Delta \xi_{1} \Delta \xi_{2} \Delta \xi_{3}}( & \delta_{1} \delta_{3}\left[\delta_{2}\left[x_{2}\right] \overline{\overline{x_{3}}}\right]-\delta_{1} \delta_{2}\left[\delta_{3}\left[x_{2}\right] \overline{\overline{x_{3}}}\right]+\delta_{2} \delta_{1}\left[\delta_{3}\left[x_{2}\right] \overline{\overline{x_{3}}}\right] \\
& \left.-\delta_{2} \delta_{3}\left[\delta_{1}\left[x_{2}\right] \overline{x_{3}}\right]+\delta_{3} \delta_{2}\left[\delta_{1}\left[x_{2}\right] \overline{x_{3}}\right]-\delta_{3} \delta_{1}\left[\delta_{2}\left[x_{2}\right] \overline{\overline{x_{3}}}\right]\right)=0
\end{aligned}
$$

since $\delta_{l}$ and $\delta_{k}$ are commutative, i.e. $\delta_{l} \delta_{k}=\delta_{k} \delta_{l}$.

The following lemma tells us that the scheme also preserves the free-stream states by integrating (3.1)-(3.2) with the same explicit SSP RK schemes [22]. 
Lemma 3.2. If the semi-discrete scheme (3.1)-(3.2) is integrated in time with the explicit SSP RK scheme from $t=t_{n}$ to $t_{n+1}=t_{n}+\Delta t_{n}$, then the resulting fully-discrete scheme preserves the free-stream states.

Proof. The forward Euler time discretization is only considered here, since the explicit SSP RK schemes are a convex combination of the forward Euler time discretizations. Assuming that $\boldsymbol{U}_{\boldsymbol{i}}^{n}=$ $\boldsymbol{U}_{0}$ is a physical constant state and the time step size is $\Delta t_{n}$, then the update of the metric Jacobian $J_{i}$ and the solution $\boldsymbol{U}_{\boldsymbol{i}}$ can be rewritten as follows

$$
\begin{aligned}
J_{\boldsymbol{i}}^{n+1}= & J_{\boldsymbol{i}}^{n}-\sum_{k=1}^{3} \frac{\Delta t_{n}}{\Delta \xi_{k}} \delta_{k}\left[J \frac{\partial \xi_{k}}{\partial t}\right]_{\boldsymbol{i}} \\
(J \boldsymbol{U})_{\boldsymbol{i}}^{n+1}= & (J \boldsymbol{U})_{\boldsymbol{i}}^{n}-\sum_{k=1}^{3} \frac{\Delta t_{n}}{\Delta \xi_{k}} \delta_{k}\left[\widehat{\boldsymbol{F}}_{k}\right]_{\boldsymbol{i}} \\
= & J_{\boldsymbol{i}}^{n} \boldsymbol{U}_{0}-\sum_{k=1}^{3} \frac{\Delta t_{n}}{\Delta \xi_{k}}\left[\left(J \frac{\partial \xi_{k}}{\partial t}\right)_{\hat{\boldsymbol{i}}_{k,+}} \boldsymbol{U}_{0}+\sum_{l=1}^{3}\left(J \frac{\partial \xi_{k}}{\partial x_{l}}\right)_{\hat{\boldsymbol{i}}_{k,+}} \boldsymbol{F}_{l}\left(\boldsymbol{U}_{0}\right)\right. \\
& \left.-\left(J \frac{\partial \xi_{k}}{\partial t}\right)_{\hat{\boldsymbol{i}}_{k,-}} \boldsymbol{U}_{0}-\sum_{l=1}^{3}\left(J \frac{\partial \xi_{k}}{\partial x_{l}}\right)_{\hat{\boldsymbol{i}}_{k,-}} \boldsymbol{F}_{l}\left(\boldsymbol{U}_{0}\right)\right] \\
= & \left(J_{\boldsymbol{i}}^{n}-\sum_{k=1}^{3} \frac{\Delta t_{n}}{\Delta \xi_{k}} \delta_{k}\left[J \frac{\partial \xi_{k}}{\partial t}\right]_{i}\right) \boldsymbol{U}_{0}-\sum_{l=1}^{3}\left(\sum_{k=1}^{3} \frac{\Delta t_{n}}{\Delta \xi_{k}} \delta_{k}\left[J \frac{\partial \xi_{k}}{\partial x_{l}}\right]_{i}\right) \boldsymbol{F}_{l}\left(\boldsymbol{U}_{0}\right) \\
= & J_{\boldsymbol{i}}^{n+1} \boldsymbol{U}_{0},
\end{aligned}
$$

where the discrete GCLs have been used in the last equality. Thus $\boldsymbol{U}_{\boldsymbol{i}}^{n+1}=(J \boldsymbol{U})_{\boldsymbol{i}}^{n+1} / J_{\boldsymbol{i}}^{n+1}=\boldsymbol{U}_{0}$. The proof is completed.

In the above proof, no specific form of the "fluxes" $\left(J \partial_{t} \xi_{k}\right)_{\hat{\boldsymbol{i}}_{k, \pm}}$ in (3.2) are given. Two suggested versions of the "fluxes" $\left(J \partial_{t} \xi_{k}\right)_{\hat{\boldsymbol{i}}_{k, \pm}}$ are presented here and compared below. It is worth noting that they do not affect the conclusion of Lemma 3.2. The first version is given by

$$
\begin{aligned}
& \left(J \frac{\partial \xi_{1}}{\partial t}\right)_{\hat{\boldsymbol{i}}_{1, \pm}}=-\sum_{l=1}^{3}\left(\dot{x}_{l}\right)_{\hat{\boldsymbol{i}}_{1, \pm}}\left(J \frac{\partial \xi_{1}}{\partial x_{l}}\right)_{\hat{\boldsymbol{i}}_{1, \pm}}, \\
& \left(J \frac{\partial \xi_{2}}{\partial t}\right)_{\hat{\boldsymbol{i}}_{2, \pm}}=-\sum_{l=1}^{3}\left(\dot{x}_{l}\right)_{\hat{\boldsymbol{i}}_{2, \pm}}\left(J \frac{\partial \xi_{2}}{\partial x_{l}}\right)_{\hat{\boldsymbol{i}}_{2, \pm}}, \\
& \left(J \frac{\partial \xi_{3}}{\partial t}\right)_{\hat{\boldsymbol{i}}_{3, \pm}}=-\sum_{l=1}^{3}\left(\dot{x}_{l}\right)_{\hat{\boldsymbol{i}}_{3, \pm}}\left(J \frac{\partial \xi_{3}}{\partial x_{l}}\right)_{\hat{\boldsymbol{i}}_{3, \pm}},
\end{aligned}
$$

where the "mesh" velocities $(\dot{\boldsymbol{x}})_{\hat{\boldsymbol{i}}_{1, \pm}},(\dot{\boldsymbol{x}})_{\hat{\boldsymbol{i}}_{2, \pm}}$, and $(\dot{\boldsymbol{x}})_{\hat{\boldsymbol{i}}_{3, \pm}}$ may be calculated by the arithmetic mean, e.g.

$$
(\dot{\boldsymbol{x}})_{i_{1}+\frac{1}{2}, i_{2}, i_{3}}=\frac{1}{4}\left[(\dot{\boldsymbol{x}})_{i_{1}+\frac{1}{2}, i_{2}-\frac{1}{2}, i_{3}-\frac{1}{2}}+(\dot{\boldsymbol{x}})_{i_{1}+\frac{1}{2}, i_{2}-\frac{1}{2}, i_{3}+\frac{1}{2}}+(\dot{\boldsymbol{x}})_{i_{1}+\frac{1}{2}, i_{2}+\frac{1}{2}, i_{3}-\frac{1}{2}}+(\dot{\boldsymbol{x}})_{i_{1}+\frac{1}{2}, i_{2}+\frac{1}{2}, i_{3}+\frac{1}{2}}\right] .
$$


Here $(\dot{\boldsymbol{x}})_{i_{1}+\frac{1}{2}, i_{2}+\frac{1}{2}, i_{3}+\frac{1}{2}}$ is the mesh velocity of the mesh point $(\boldsymbol{x})_{i_{1}+\frac{1}{2}, i_{2}+\frac{1}{2}, i_{3}+\frac{1}{2}}$, which will be provided by solving the mesh equations in Section 4. Combining (3.8) with (3.2) gives our first semi-discrete VCL, denoted by VCL1, which is easy to be implemented.

The second version of the "fluxes" $\left(J \partial_{t} \xi_{k}\right)_{\hat{\boldsymbol{i}}_{k, \pm}}$ is based on the reformulations of the Jacobian and the temporal metrics [1] as follows

$$
\begin{aligned}
J= & \frac{\partial}{\partial \xi_{3}}\left\{\left[\frac{\partial}{\partial \xi_{2}}\left(\frac{\partial x_{1}}{\partial \xi_{1}} x_{2}\right)-\frac{\partial}{\partial \xi_{1}}\left(\frac{\partial x_{1}}{\partial \xi_{2}} x_{2}\right)\right] x_{3}\right\}+\frac{\partial}{\partial \xi_{2}}\left\{\left[\frac{\partial}{\partial \xi_{1}}\left(\frac{\partial x_{1}}{\partial \xi_{3}} x_{2}\right)-\frac{\partial}{\partial \xi_{3}}\left(\frac{\partial x_{1}}{\partial \xi_{1}} x_{2}\right)\right] x_{3}\right\} \\
& +\frac{\partial}{\partial \xi_{1}}\left\{\left[\frac{\partial}{\partial \xi_{3}}\left(\frac{\partial x_{1}}{\partial \xi_{2}} x_{2}\right)-\frac{\partial}{\partial \xi_{2}}\left(\frac{\partial x_{1}}{\partial \xi_{3}} x_{2}\right)\right] x_{3}\right\}, \\
J \frac{\partial \xi_{1}}{\partial t}= & \frac{\partial}{\partial \xi_{2}}\left\{\left[\frac{\partial}{\partial \xi_{3}}\left(\frac{\partial x_{1}}{\partial t} x_{2}\right)-\frac{\partial}{\partial t}\left(\frac{\partial x_{1}}{\partial \xi_{3}} x_{2}\right)\right] x_{3}\right\}+\frac{\partial}{\partial \xi_{3}}\left\{\left[\frac{\partial}{\partial t}\left(\frac{\partial x_{1}}{\partial \xi_{2}} x_{2}\right)-\frac{\partial}{\partial \xi_{2}}\left(\frac{\partial x_{1}}{\partial t} x_{2}\right)\right] x_{3}\right\} \\
& +\frac{\partial}{\partial t}\left\{\left[\frac{\partial}{\partial \xi_{2}}\left(\frac{\partial x_{1}}{\partial \xi_{3}} x_{2}\right)-\frac{\partial}{\partial \xi_{3}}\left(\frac{\partial x_{1}}{\partial \xi_{2}} x_{2}\right)\right] x_{3}\right\}, \\
J \frac{\partial \xi_{2}}{\partial t}= & \frac{\partial}{\partial \xi_{3}}\left\{\left[\frac{\partial}{\partial \xi_{1}}\left(\frac{\partial x_{1}}{\partial t} x_{2}\right)-\frac{\partial}{\partial t}\left(\frac{\partial x_{1}}{\partial \xi_{1}} x_{2}\right)\right] x_{3}\right\}+\frac{\partial}{\partial t}\left\{\left[\frac{\partial}{\partial \xi_{3}}\left(\frac{\partial x_{1}}{\partial \xi_{1}} x_{2}\right)-\frac{\partial}{\partial \xi_{1}}\left(\frac{\partial x_{1}}{\partial \xi_{3}} x_{2}\right)\right] x_{3}\right\} \\
& +\frac{\partial}{\partial \xi_{1}}\left\{\left[\frac{\partial}{\partial t}\left(\frac{\partial x_{1}}{\partial \xi_{3}} x_{2}\right)-\frac{\partial}{\partial \xi_{3}}\left(\frac{\partial x_{1}}{\partial t} x_{2}\right)\right] x_{3}\right\}, \\
J \frac{\partial \xi_{3}}{\partial t}= & \frac{\partial}{\partial t}\left\{\left[\frac{\partial}{\partial \xi_{1}}\left(\frac{\partial x_{1}}{\partial \xi_{2}} x_{2}\right)-\frac{\partial}{\partial \xi_{2}}\left(\frac{\partial x_{1}}{\partial \xi_{1}} x_{2}\right)\right] x_{3}\right\}+\frac{\partial}{\partial \xi_{1}}\left\{\left[\frac{\partial}{\partial \xi_{2}}\left(\frac{\partial x_{1}}{\partial t} x_{2}\right)-\frac{\partial}{\partial t}\left(\frac{\partial x_{1}}{\partial \xi_{2}} x_{2}\right)\right] x_{3}\right\} \\
& +\frac{\partial}{\partial \xi_{2}}\left\{\left[\frac{\partial}{\partial t}\left(\frac{\partial x_{1}}{\partial \xi_{1}} x_{2}\right)-\frac{\partial}{\partial \xi_{1}}\left(\frac{\partial x_{1}}{\partial t} x_{2}\right)\right] x_{3}\right\},
\end{aligned}
$$

and the second-order central difference and average approximated the spatial derivatives, similar to (3.7), thus the VCL in (3.2) is approximated in space by

$$
\begin{aligned}
\frac{\mathrm{d} J_{i}}{\mathrm{~d} t}= & -\sum_{l=1}^{3} \frac{1}{\Delta \xi_{k}} \delta_{k}\left[J \frac{\partial \xi_{k}}{\partial t}\right]_{i} \\
= & -\frac{1}{\Delta \xi_{1}} \delta_{1}\left[\frac{\partial}{\partial t}\left\{\left[\frac{\partial}{\partial \xi_{2}}\left(\frac{\partial x_{1}}{\partial \xi_{3}} x_{2}\right)-\frac{\partial}{\partial \xi_{3}}\left(\frac{\partial x_{1}}{\partial \xi_{2}} x_{2}\right)\right] x_{3}\right\}\right]_{i} \\
& -\frac{1}{\Delta \xi_{2}} \delta_{2}\left[\frac{\partial}{\partial t}\left\{\left[\frac{\partial}{\partial \xi_{3}}\left(\frac{\partial x_{1}}{\partial \xi_{1}} x_{2}\right)-\frac{\partial}{\partial \xi_{1}}\left(\frac{\partial x_{1}}{\partial \xi_{3}} x_{2}\right)\right] x_{3}\right\}\right]_{i} \\
& -\frac{1}{\Delta \xi_{3}} \delta_{3}\left[\frac{\partial}{\partial t}\left\{\left[\frac{\partial}{\partial \xi_{1}}\left(\frac{\partial x_{1}}{\partial \xi_{2}} x_{2}\right)-\frac{\partial}{\partial \xi_{2}}\left(\frac{\partial x_{1}}{\partial \xi_{1}} x_{2}\right)\right] x_{3}\right\}\right]_{i} \\
= & :-\frac{1}{\Delta \xi_{1}} \delta_{1}\left[\frac{\partial}{\partial t} A_{1}\right]_{i}-\frac{1}{\Delta \xi_{2}} \delta_{2}\left[\frac{\partial}{\partial t} A_{2}\right]_{i}-\frac{1}{\Delta \xi_{3}} \delta_{3}\left[\frac{\partial}{\partial t} A_{3}\right]_{i},
\end{aligned}
$$

which gives our second semi-discrete VCL, denoted by VCL2. Obviously, it requires more operation, but it can well approach to the value of the Jacobian $J$ calculated by the first equation of (3.9). In fact, if the mesh trajectories are assumed to be linear in time as follows

$$
\boldsymbol{x}(t)=\frac{t_{n+1}-t}{\Delta t_{n}} \boldsymbol{x}^{n}+\frac{t-t_{n}}{\Delta t_{n}} \boldsymbol{x}^{n+1}, t \in\left[t_{n}, t_{n+1}\right], \Delta t_{n}=t_{n+1}-t_{n},
$$

inspired by [43], then the terms $A_{k}, k=1,2,3$ are cubic polynomials of $t$, so that $\frac{\partial}{\partial t} A_{k}$ is a quadratic 
polynomial of $t$, which can be expressed as

$$
\begin{aligned}
\frac{\partial}{\partial t} A_{k}= & \frac{1}{2\left(\Delta t_{n}\right)^{3}}\left[\left(\Delta t_{n}\right)^{2}\left(-11 A_{k}^{n}+18 A_{k}^{n+\frac{1}{3}}-9 A_{k}^{n+\frac{2}{3}}+2 A_{k}^{n+1}\right)\right. \\
& +18 \Delta t_{n}\left(2 A_{k}^{n}-5 A_{k}^{n+\frac{1}{3}}+4 A_{k}^{n+\frac{2}{3}}-A_{k}^{n+1}\right)\left(t-t_{n}\right) \\
& \left.-27\left(A_{k}^{n}-3 A_{k}^{n+\frac{1}{3}}+3 A_{k}^{n+\frac{2}{3}}-A_{k}^{n+1}\right)\left(t-t_{n}\right)^{2}\right],
\end{aligned}
$$

where the superscript denotes the value at corresponding time level. If following the first equation in (3.9) to compute $J$ at time $t_{m}$ by

$$
J_{i}^{m}=-\sum_{k=1}^{3} \frac{1}{\Delta \xi_{k}} \delta_{k}\left[A_{k}^{m}\right]_{i}
$$

then substituting (3.12) into (3.10) and using (3.13) gives

$$
\begin{aligned}
\frac{\mathrm{d} J}{\mathrm{~d} t}= & \frac{1}{2\left(\Delta t_{n}\right)^{3}}\left[\left(\Delta t_{n}\right)^{2}\left(-11 J^{n}+18 J^{n+\frac{1}{3}}-9 J^{n+\frac{2}{3}}+2 J^{n+1}\right)\right. \\
& +18 \Delta t_{n}\left(2 J^{n}-5 J^{n+\frac{1}{3}}+4 J^{n+\frac{2}{3}}-J^{n+1}\right)\left(t-t_{n}\right) \\
& \left.-27\left(J^{n}-3 J^{n+\frac{1}{3}}+3 J^{n+\frac{2}{3}}-J^{n+1}\right)\left(t-t_{n}\right)^{2}\right] .
\end{aligned}
$$

Here $J^{n}, J^{n+\frac{1}{3}}, J^{n+\frac{2}{3}}, J^{n+1}$ are known, so that (3.14) is a linear ordinary differential equation (ODE) with the RHS of a quadratic polynomial of $t$. If the third-order SSP RK method is used to integrate (3.14), then it will hold exactly. In the $2 \mathrm{D}$ case, it will be a linear ODE with the RHS of a linear polynomial of $t$, so the second-order SSP RK method is enough.

Remark 3.2. In the $3 D$ case, the second-order SSP RK method can approximate (3.2) to the second-order accuracy and no obvious difference of the solutions is found between the SSP secondand third-order and RK methods, see the numerical results in Section 5.

\subsection{EC flux}

What follows is to find an EC flux satisfying (3.4). For the second-order accurate scheme, we choose the EC flux as follows

$$
\widetilde{\boldsymbol{F}}_{k, \hat{\boldsymbol{i}}_{k, \pm}}=\left(J \frac{\partial \xi_{k}}{\partial t}\right)_{\hat{\boldsymbol{i}}_{k, \pm}} \widetilde{\boldsymbol{U}}_{\hat{\boldsymbol{i}}_{k, \pm}}^{\mathrm{RHD}}+\sum_{l=1}^{3}\left(J \frac{\partial \xi_{k}}{\partial x_{l}}\right)_{\hat{\boldsymbol{i}}_{k, \pm}} \widetilde{\boldsymbol{F}}_{l, \hat{\boldsymbol{i}}_{k, \pm}}^{\mathrm{RHD}},
$$

where $\widetilde{\boldsymbol{F}}_{l, \hat{\boldsymbol{i}}_{k, \pm}}^{\mathrm{RHD}}$ is the EC flux of the RHD equations on the static mesh satisfying

$$
\llbracket \boldsymbol{V} \rrbracket_{\hat{\boldsymbol{i}}_{k, \pm}}^{\mathrm{T}} \widetilde{\boldsymbol{F}}_{l, \hat{\boldsymbol{i}}_{k, \pm}}^{\mathrm{RHD}}=\llbracket \psi_{l} \rrbracket_{\hat{\boldsymbol{i}}_{k, \pm}}
$$


and $\widetilde{\boldsymbol{U}}_{\hat{\boldsymbol{i}}_{k, \pm}}^{\mathrm{RHD}}$ is obtained by the same procedure in [13] satisfying

$$
\llbracket \boldsymbol{V} \rrbracket_{\hat{\boldsymbol{i}}_{k, \pm}}^{\mathrm{T}} \widetilde{\boldsymbol{U}}_{\hat{\boldsymbol{i}}_{k, \pm}}^{\mathrm{RHD}}=\llbracket \phi \rrbracket_{\hat{\boldsymbol{i}}_{k, \pm}} .
$$

For example, the specific expressions of $\widetilde{\boldsymbol{F}}_{1}^{\mathrm{RHD}}$ and $\widetilde{\boldsymbol{U}}^{\mathrm{RHD}}$ are respectively given as follows

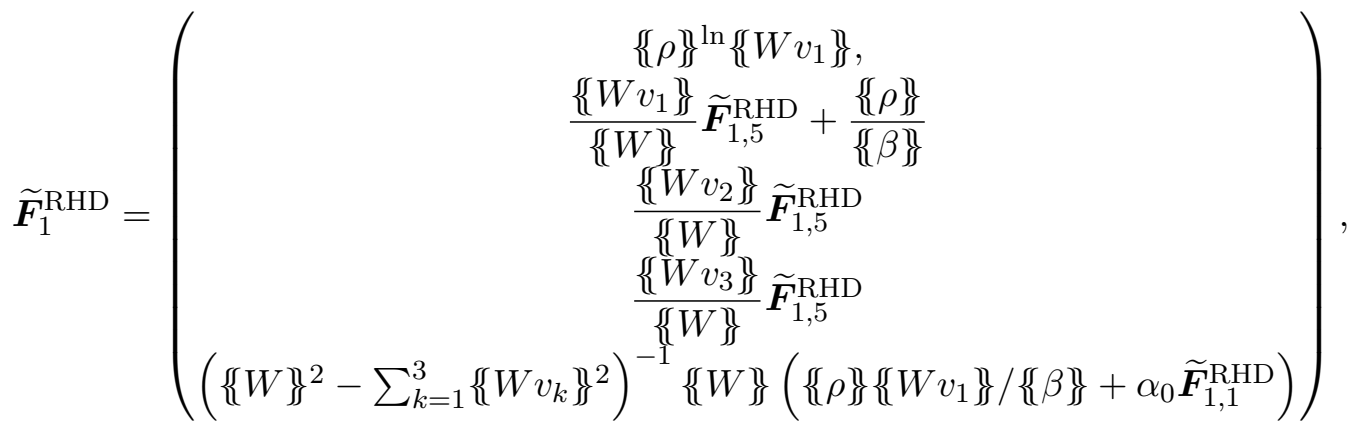

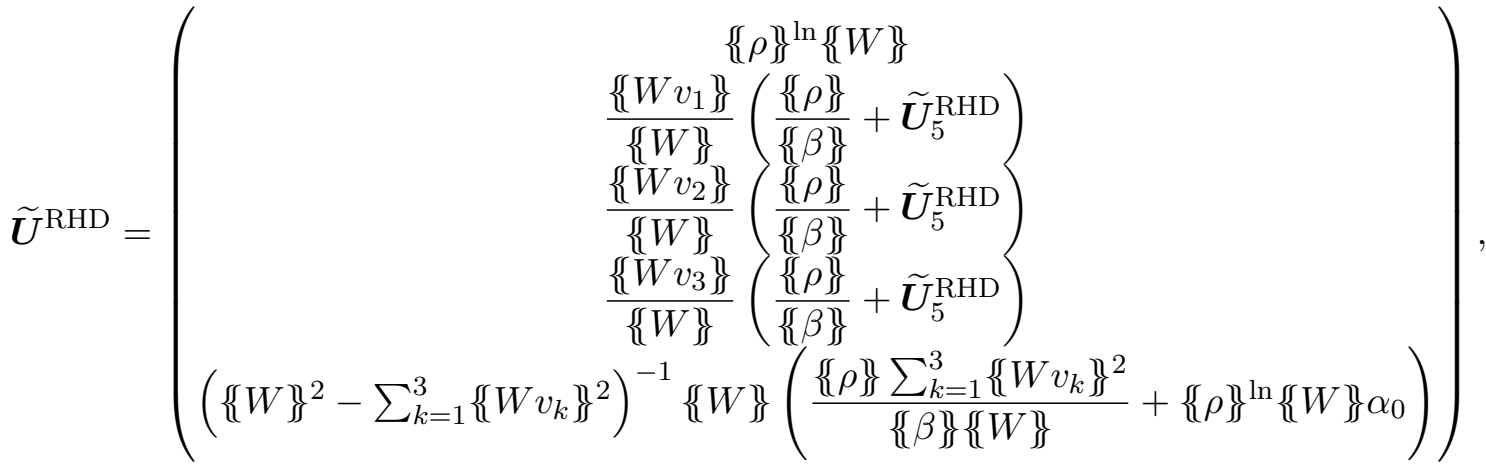

where $\left\{\{a\}^{\ln }=\llbracket a \rrbracket / \llbracket \ln a \rrbracket\right.$ is the logarithmic mean, see $[29], \alpha_{0}=1+1 /(\Gamma-1) /\left\{\{\beta\}^{\ln }, \beta=\rho / p\right.$, and $\widetilde{\boldsymbol{F}}_{1,5}^{\mathrm{RHD}}$ and $\widetilde{\boldsymbol{U}}_{5}^{\mathrm{RHD}}$ denote the 5 -th component of $\widetilde{\boldsymbol{F}}_{1}^{\mathrm{RHD}}$ and $\widetilde{\boldsymbol{U}}^{\mathrm{RHD}}$, respectively.

\subsection{ES schemes}

It is known that for the quasi-linear hyperbolic conservation laws, the entropy identity is available only if the solution is smooth. In other words, the entropy is not conserved if the discontinuities such as the shock waves appear in the solution. Moreover, the EC scheme may produce serious nonphysical oscillations near the discontinuities. Those motivate us to develop the ES scheme (satisfying the entropy inequality for the given entropy pair) in this section by adding a suitable dissipation term to the EC flux (3.15).

Following [46], adding a dissipation term to the EC flux $\widetilde{\boldsymbol{F}}_{k, \hat{\boldsymbol{i}}_{k, \pm}}$ gives the ES flux

$$
\widehat{\boldsymbol{F}}_{k, \hat{\boldsymbol{i}}_{k, \pm}}=\widetilde{\boldsymbol{F}}_{k, \hat{\boldsymbol{i}}_{k, \pm}}-\frac{1}{2} \boldsymbol{D}_{\hat{\boldsymbol{i}}_{k, \pm}} \llbracket \boldsymbol{V} \rrbracket_{\hat{\boldsymbol{i}}_{k, \pm}},
$$

satisfying

$$
\llbracket \boldsymbol{V} \rrbracket_{\hat{\boldsymbol{i}}_{k, \pm}}^{\mathrm{T}} \cdot \widehat{\boldsymbol{F}}_{k, \hat{\boldsymbol{i}}_{k, \pm}}-\left(J \frac{\partial \xi_{k}}{\partial t}\right)_{\hat{\boldsymbol{i}}_{k, \pm}} \llbracket \phi \rrbracket_{\hat{\boldsymbol{i}}_{k, \pm}}-\sum_{l=1}^{3}\left(J \frac{\partial \xi_{k}}{\partial x_{l}}\right)_{\hat{\boldsymbol{i}}_{k, \pm}} \llbracket \psi_{l} \rrbracket_{\hat{\boldsymbol{i}}_{k, \pm}} \leqslant 0
$$


where $\boldsymbol{D}_{\hat{\boldsymbol{i}}_{k, \pm}}$ is a symmetric positive semi-definite matrix. It is easy to prove that the scheme (3.1)(3.2) with the numerical flux (3.16) is ES, that is, it satisfies the semi-discrete entropy inequality

$$
\frac{\mathrm{d}}{\mathrm{d} t} J_{\boldsymbol{i}} \eta\left(\boldsymbol{U}_{\boldsymbol{i}}(t)\right)+\sum_{k=1}^{3} \frac{1}{\Delta \xi_{k}} \delta_{k}\left[\widehat{q}_{k}(t)\right]_{\boldsymbol{i}} \leqslant 0,
$$

with the numerical entropy flux

$$
\widehat{q}_{k, \hat{\boldsymbol{i}}_{k, \pm}}=\widetilde{q}_{k, \hat{\boldsymbol{i}}_{k, \pm}}-\frac{1}{2}\{\boldsymbol{V}\}_{\hat{\boldsymbol{i}}_{k, \pm}} \boldsymbol{D}_{\hat{\boldsymbol{i}}_{k, \pm}} \llbracket \boldsymbol{V} \rrbracket_{\hat{\boldsymbol{i}}_{k, \pm}},
$$

being consistent with the continuous entropy flux $J \frac{\partial \xi_{k}}{\partial t} \eta+\sum_{l=1}^{3} J \frac{\partial \xi_{k}}{\partial x_{l}} q_{l}$.

Let us give a choice of $\boldsymbol{D}_{\hat{\boldsymbol{i}}_{k, \pm}}$ in the ES flux (3.16). According to [39], there exists a set of scaled eigenvectors $\boldsymbol{R}$ such that

$$
\frac{\partial \boldsymbol{U}}{\partial \boldsymbol{V}}=\boldsymbol{R} \boldsymbol{R}^{\mathrm{T}}, \frac{\partial \boldsymbol{F}_{1}}{\partial \boldsymbol{U}}=\boldsymbol{R} \boldsymbol{\Lambda} \boldsymbol{R}^{-1}, \boldsymbol{\Lambda}=\operatorname{diag}\left\{\lambda_{1}, \ldots, \lambda_{5}\right\}
$$

where the eigenvalues $\lambda_{1}, \ldots, \lambda_{5}$ are given by

$$
\lambda_{1}=\lambda_{-}, \lambda_{5}=\lambda_{+}, \lambda_{\ell}=v_{1}, \ell=2,3,4, \lambda_{ \pm}=\frac{v_{1}\left(1-c_{s}^{2}\right) \pm c_{s} / W \sqrt{1-v_{1}^{2}-\left(|\boldsymbol{v}|^{2}-v_{1}^{2}\right) c_{s}^{2}}}{1-|\boldsymbol{v}|^{2} c_{s}^{2}},
$$

and

$$
\begin{aligned}
& \boldsymbol{R}=\left[\begin{array}{ccccc}
1 & 1 / W & W v_{2} & W v_{3} & 1 \\
h W \mathcal{A}_{-} \lambda_{-} & v_{1} & 2 h W^{2} v_{1} v_{2} & 2 h W^{2} v_{1} v_{3} & h W \mathcal{A}_{+} \lambda_{+} \\
h W v_{2} & v_{2} & h\left(1+2 W^{2} v_{2}^{2}\right) & 2 h W^{2} v_{2} v_{3} & h W v_{2} \\
h W v_{3} & v_{3} & 2 h W^{2} v_{2} v_{3} & h\left(1+2 W^{2} v_{3}^{2}\right) & h W v_{3} \\
h W \mathcal{A}_{-} & 1 & 2 h W^{2} v_{2} & 2 h W^{2} v_{3} & h W \mathcal{A}_{+}
\end{array}\right] \\
& \times\left[\begin{array}{ccccc}
\sqrt{\frac{\mathcal{B}-\mathcal{C}}{2}} & 0 & 0 & 0 & 0 \\
0 & \sqrt{\frac{(\Gamma-1) \rho W^{3}}{\Gamma}} & 0 & 0 & 0 \\
0 & 0 & \sqrt{\frac{p W\left(1-v_{1}^{2}-v_{2}^{2}\right)}{h\left(1-v_{1}^{2}\right)}} & 0 & 0 \\
0 & 0 & -v_{2} v_{3} \sqrt{\frac{p W}{h\left(1-v_{1}^{2}\right)\left(1-v_{1}^{2}-v_{2}^{2}\right)}} & \sqrt{\frac{p}{h W\left(1-v_{1}^{2}-v_{2}^{2}\right)}} & 0 \\
0 & 0 & 0 & 0 & \sqrt{\frac{\mathcal{B}+\mathcal{C}}{2}}
\end{array}\right],
\end{aligned}
$$

here $\mathcal{A}_{ \pm}=\frac{1-v_{1}^{2}}{1-v_{1} \lambda_{ \pm}}, \mathcal{B}=\frac{\rho W\left(1-v_{1}^{2}-\left(|\boldsymbol{v}|^{2}-v_{1}^{2}\right) c_{s}^{2}\right)}{\Gamma\left(1-v_{1}^{2}\right)}, \mathcal{C}=\frac{\rho v_{1} c_{s} \sqrt{1-v_{1}^{2}-\left(|\boldsymbol{v}|^{2}-v_{1}^{2}\right) c_{s}^{2}}}{\Gamma\left(1-v_{1}^{2}\right)} . \quad$ Using the rotational invariance gives

$$
\begin{aligned}
& \partial\left(J \frac{\partial \xi_{k}}{\partial t} \boldsymbol{U}+\sum_{l=1}^{3} J \frac{\partial \xi_{k}}{\partial x_{l}} \boldsymbol{F}_{l}\right) / \partial \boldsymbol{U} \\
= & \partial\left(J \frac{\partial \xi_{k}}{\partial t} \boldsymbol{U}+L_{k} \boldsymbol{T}^{-1} \boldsymbol{F}_{1}(\boldsymbol{T} \boldsymbol{U})\right) / \partial \boldsymbol{U}
\end{aligned}
$$




$$
\begin{aligned}
& =J \frac{\partial \xi_{k}}{\partial t} \boldsymbol{I}+L_{k} \boldsymbol{T}^{-1} \boldsymbol{R}(\boldsymbol{T} \boldsymbol{U}) \boldsymbol{\Lambda}(\boldsymbol{T} \boldsymbol{U}) \boldsymbol{R}^{-1}(\boldsymbol{T} \boldsymbol{U}) \boldsymbol{T} \\
& =\boldsymbol{T}^{-1} \boldsymbol{R}(\boldsymbol{T} \boldsymbol{U})\left(J \frac{\partial \xi_{k}}{\partial t} \boldsymbol{I}+L_{k} \boldsymbol{\Lambda}(\boldsymbol{T} \boldsymbol{U})\right) \boldsymbol{R}^{-1}(\boldsymbol{T} \boldsymbol{U}) \boldsymbol{T}
\end{aligned}
$$

where $L_{k}=\sqrt{\sum_{l=1}^{3}\left(J \frac{\partial \xi_{k}}{\partial x_{l}}\right)^{2}}$, and $\boldsymbol{T}$ denotes the "rotational" matrix defined by

$$
\begin{aligned}
& \boldsymbol{T}=\left[\begin{array}{ccccc}
1 & 0 & 0 & 0 & 0 \\
0 & \cos \varphi \cos \theta & \cos \varphi \sin \theta & \sin \varphi & 0 \\
0 & -\sin \theta & \cos \theta & 0 & 0 \\
0 & -\sin \varphi \cos \theta & -\sin \varphi \sin \theta & \cos \varphi & 0 \\
0 & 0 & 0 & 0 & 1
\end{array}\right], \\
& \theta=\arctan \left(\left(J \frac{\partial \xi_{k}}{\partial x_{2}}\right) /\left(J \frac{\partial \xi_{k}}{\partial x_{1}}\right)\right), \\
& \varphi=\arctan \left(\left(J \frac{\partial \xi_{k}}{\partial x_{3}}\right) / \sqrt{\left(J \frac{\partial \xi_{k}}{\partial x_{1}}\right)^{2}+\left(J \frac{\partial \xi_{k}}{\partial x_{2}}\right)^{2}}\right)
\end{aligned}
$$

Then following the dissipation term in the Roe scheme yields

$$
\begin{aligned}
& -\frac{1}{2} \boldsymbol{T}^{-1} \boldsymbol{R}(\boldsymbol{T U})\left|J \frac{\partial \xi_{k}}{\partial t} \boldsymbol{I}+L_{k} \boldsymbol{\Lambda}(\boldsymbol{T} \boldsymbol{U})\right| \boldsymbol{R}^{-1}(\boldsymbol{T U}) \boldsymbol{T} \llbracket \boldsymbol{U} \rrbracket \\
& =-\frac{1}{2} \boldsymbol{T}^{-1} \boldsymbol{R}(\boldsymbol{T} \boldsymbol{U})\left|J \frac{\partial \xi_{k}}{\partial t} \boldsymbol{I}+L_{k} \boldsymbol{\Lambda}(\boldsymbol{T} \boldsymbol{U})\right| \boldsymbol{R}^{-1}(\boldsymbol{T} \boldsymbol{U}) \llbracket \boldsymbol{T U} \rrbracket \\
& \approx-\frac{1}{2} \boldsymbol{T}^{-1} \boldsymbol{R}(\boldsymbol{T} \boldsymbol{U})\left|J \frac{\partial \xi_{k}}{\partial t} \boldsymbol{I}+L_{k} \boldsymbol{\Lambda}(\boldsymbol{T} \boldsymbol{U})\right| \boldsymbol{R}^{-1}(\boldsymbol{T} \boldsymbol{U}) \boldsymbol{R}(\boldsymbol{T} \boldsymbol{U}) \boldsymbol{R}^{\mathrm{T}}(\boldsymbol{T} \boldsymbol{U}) \boldsymbol{T} \llbracket \boldsymbol{V} \rrbracket \\
& =-\frac{1}{2} \boldsymbol{T}^{-1} \boldsymbol{R}(\boldsymbol{T} \boldsymbol{U})\left|J \frac{\partial \xi_{k}}{\partial t} \boldsymbol{I}+L_{k} \boldsymbol{\Lambda}(\boldsymbol{T U})\right| \boldsymbol{R}^{\mathrm{T}}(\boldsymbol{T U}) \boldsymbol{T} \llbracket \boldsymbol{V} \rrbracket .
\end{aligned}
$$

Based on that, the matrix $\boldsymbol{D}_{\hat{\boldsymbol{i}}_{k, \pm}}$ in (3.16) can be chosen as follows (evaluated at the interface point $\left.\hat{\boldsymbol{i}}_{k, \pm}\right)$

$$
\boldsymbol{D}=\boldsymbol{T}^{-1} \boldsymbol{R}(\boldsymbol{T} \boldsymbol{U})\left|J \frac{\partial \xi_{k}}{\partial t} \boldsymbol{I}+L_{k} \boldsymbol{\Lambda}(\boldsymbol{T} \boldsymbol{U})\right| \boldsymbol{R}^{\mathrm{T}}(\boldsymbol{T} \boldsymbol{U}) \boldsymbol{T}
$$

where the matrix $\left|J \frac{\partial \xi_{k}}{\partial t} \boldsymbol{I}+L_{k} \boldsymbol{\Lambda}(\boldsymbol{T U})\right|$ is taken as

$$
\left|J \frac{\partial \xi_{k}}{\partial t} \boldsymbol{I}+L_{k} \boldsymbol{\Lambda}(\boldsymbol{T U})\right|:=\max \left\{\left|J \frac{\partial \xi_{k}}{\partial t}+L_{k} \lambda_{1}(\boldsymbol{T U})\right|, \ldots,\left|J \frac{\partial \xi_{k}}{\partial t}+L_{k} \lambda_{5}(\boldsymbol{T} \boldsymbol{U})\right|\right\} \boldsymbol{I},
$$

and the values of $\left|J \frac{\partial \xi_{k}}{\partial t} \boldsymbol{I}+L_{k} \boldsymbol{\Lambda}(\boldsymbol{T} \boldsymbol{U})\right|_{\hat{\boldsymbol{i}}_{k, \pm}}$ and $\boldsymbol{R}_{\hat{\boldsymbol{i}}_{k, \pm}}(\boldsymbol{T} \boldsymbol{U})$ are calculated by using the arithmetic mean values of the left and right states.

To obtain a second-order accurate ES scheme, the dissipation term in (3.16) has to be improved. Here the second-order TVD reconstruction is performed in the scaled entropy variables $\boldsymbol{w}=\boldsymbol{R}^{\mathrm{T}} \boldsymbol{V}$. 
More specifically, the linear reconstruction of $\boldsymbol{w}$ with the minmod limiter is used in the $i_{k}$-direction to obtain the left and right limit values at $\hat{\boldsymbol{i}}_{k,+}$, denoted by $\boldsymbol{w}_{\hat{\boldsymbol{i}}_{k,+}}^{-}$and $\boldsymbol{w}_{\hat{\boldsymbol{i}}_{k,+}}^{+}$, and then to define

$$
\langle\langle\boldsymbol{w}\rangle\rangle_{\hat{\boldsymbol{i}}_{k,+}}=\boldsymbol{w}_{\hat{\boldsymbol{i}}_{k,+}}^{+}-\boldsymbol{w}_{\hat{\boldsymbol{i}}_{k,+}^{-}}^{-}
$$

Because of the "sign" property

$$
\left.\operatorname{sign}(\langle\boldsymbol{w}\rangle\rangle_{\hat{\boldsymbol{i}}_{k,+}}\right)=\operatorname{sign}\left(\llbracket \boldsymbol{w} \rrbracket_{\hat{\boldsymbol{i}}_{k,+}}\right) \text {, }
$$

utilizing the reconstructed jump in the dissipation term can give the following second-order ES scheme

$$
\frac{\mathrm{d}}{\mathrm{d} t}(J \boldsymbol{U})_{i}=-\sum_{k=1}^{3} \frac{1}{\Delta \xi_{k}} \delta_{k}\left[\widehat{\boldsymbol{F}}_{k}^{2 \mathrm{nd}}\right]_{\boldsymbol{i}},
$$

where

$$
\widehat{\boldsymbol{F}}_{k, \hat{\boldsymbol{i}}_{k, \pm}}^{2 \mathrm{nd}}=\widetilde{\boldsymbol{F}}_{k, \hat{\boldsymbol{i}}_{k, \pm}}-\frac{1}{2} \boldsymbol{D}_{\hat{\boldsymbol{i}}_{k, \pm}}\langle\langle\boldsymbol{w}\rangle\rangle_{\hat{\boldsymbol{i}}_{k, \pm}}
$$

Remark 3.3. The ES schemes preserve the free-stream states since the dissipation terms are given by using the jump of the entropy variables, which vanish as the solution is a constant state.

\section{Adaptive moving mesh strategy}

This section presents our adaptive moving mesh strategy at time $t=t_{n}$, but focus on the mesh iteration redistribution. The dependence of the variables on $t$ will be omitted, unless otherwise stated. Consider the following mesh adaption functional

$$
\widetilde{E}(\boldsymbol{x})=\frac{1}{2} \sum_{k=1}^{3} \int_{\Omega_{l}}\left(\nabla_{\boldsymbol{\xi}} x_{k}\right)^{\mathrm{T}} \boldsymbol{G}_{k}\left(\nabla_{\boldsymbol{\xi}} x_{k}\right) \mathrm{d} \boldsymbol{\xi},
$$

where $\boldsymbol{G}_{k}$ is the given symmetric positive definite matrix, depending on the solution $\boldsymbol{U}$. More terms can be added to the above functional to control other aspects of the mesh such as the orthogonality and the alignment with a given vector field, see e.g. [4, 5, 28]. Solving the Euler-Lagrange equations of $(4.1)$

$$
\nabla_{\boldsymbol{\xi}} \cdot\left(\boldsymbol{G}_{k} \nabla_{\boldsymbol{\xi}} x_{k}\right)=0, \boldsymbol{\xi} \in \Omega_{c}, k=1,2,3,
$$

will give directly a coordinate transformation $\boldsymbol{x}=\boldsymbol{x}(\boldsymbol{\xi})$ from the computational domain $\Omega_{c}$ to the physical domain $\Omega_{p}$.

The concentration of the mesh points is controlled by $\boldsymbol{G}_{k}$, which in general depends on the solutions or their derivatives of the underlying governing equations and is one of the most important elements in the adaptive moving mesh method. Different problems may be equipped with different $\boldsymbol{G}_{k}$. Following the Winslow variable diffusion method [56], the simplest choice of $\boldsymbol{G}_{k}$ is

$$
\boldsymbol{G}_{k}=\omega \boldsymbol{I}_{3}
$$


where $\omega$ is a positive weight function, called the monitor function. For example, $\omega$ can be taken as

$$
\omega=\sqrt{1+\alpha\left|\nabla_{\boldsymbol{\xi}} \sigma\right| / \max \left|\nabla_{\boldsymbol{\xi}} \sigma\right|}
$$

where $\sigma$ is some physical variable and $\alpha>0$ is a parameter. There are several other choices of the monitor functions, see [7, 23, 24, 48, 50].

Remark 4.1. The monitor function is computed from the solutions of the underlying physical equations, thus is not smooth in general. To get a smoother (adaptive) mesh, the following low pass filter

$$
\omega_{i_{1}, i_{2}, i_{3}} \leftarrow \sum_{j_{1}, j_{2}, j_{3}=0, \pm 1}\left(\frac{1}{2}\right)^{\left|j_{1}\right|+\left|j_{2}\right|+\left|j_{3}\right|+3} \omega_{i_{1}+j_{1}, i_{2}+j_{2}, i_{3}+j_{3}}
$$

is applied $2 \sim 3$ times in this work.

The mesh equations (4.2) are approximated by the central difference scheme on the computational mesh and then solved by using the Jacobi iteration method

$$
\begin{aligned}
& \omega_{i_{1}+1, i_{2}+\frac{1}{2}, i_{3}+\frac{1}{2}}\left(\boldsymbol{x}_{i_{1}+\frac{3}{2}, i_{2}+\frac{1}{2}, i_{3}+\frac{1}{2}}^{[\nu]}-\boldsymbol{x}_{i_{1}+\frac{1}{2}, i_{2}+\frac{1}{2}, i_{3}+\frac{1}{2}}^{[\nu+1]}\right) \\
- & \omega_{i_{1}, i_{2}+\frac{1}{2}, i_{3}+\frac{1}{2}}\left(\boldsymbol{x}_{i_{1}+\frac{1}{2}, i_{2}+\frac{1}{2}, i_{3}+\frac{1}{2}}^{[\nu+1]}-\boldsymbol{x}_{i_{1}-\frac{1}{2}, i_{2}+\frac{1}{2}, i_{3}+\frac{1}{2}}^{[\nu]}\right) \\
+ & \omega_{i_{1}+\frac{1}{2}, i_{2}+1, i_{3}+\frac{1}{2}}\left(\boldsymbol{x}_{i_{1}+\frac{1}{2}, i_{2}+\frac{3}{2}, i_{3}+\frac{1}{2}}^{[\nu]}-\boldsymbol{x}_{i_{1}+\frac{1}{2}, i_{2}+\frac{1}{2}, i_{3}+\frac{1}{2}}^{[\nu+1]}\right) \\
- & \omega_{i_{1}+\frac{1}{2}, i_{2}, i_{3}+\frac{1}{2}}\left(\boldsymbol{x}_{i_{1}+\frac{1}{2}, i_{2}+\frac{1}{2}, i_{3}+\frac{1}{2}}^{[\nu+1]}-\boldsymbol{x}_{i_{1}+\frac{1}{2}, i_{2}-\frac{1}{2}, i_{3}+\frac{1}{2}}^{[\nu]}\right) \\
+ & \omega_{i_{1}+\frac{1}{2}, i_{2}+\frac{1}{2}, i_{3}+1}\left(\boldsymbol{x}_{i_{1}+\frac{1}{2}, i_{2}+\frac{1}{2}, i_{3}+\frac{3}{2}}^{[\nu]}-\boldsymbol{x}_{i_{1}+\frac{1}{2}, i_{2}+\frac{1}{2}, i_{3}+\frac{1}{2}}^{[\nu+1]}\right) \\
- & \omega_{i_{1}+\frac{1}{2}, i_{2}+\frac{1}{2}, i_{3}}\left(\boldsymbol{x}_{i_{1}+\frac{1}{2}, i_{2}+\frac{1}{2}, i_{3}+\frac{1}{2}}^{[\nu+1]}-\boldsymbol{x}_{i_{1}+\frac{1}{2}, i_{2}+\frac{1}{2}, i_{3}-\frac{1}{2}}^{[\nu]}\right)=0, \nu=0,1, \cdots, \mu
\end{aligned}
$$

in parallel, where $\boldsymbol{x}_{i_{1}+\frac{1}{2}, i_{2}+\frac{1}{2}, i_{3}+\frac{1}{2}}^{[0]}:=\boldsymbol{x}_{i_{1}+\frac{1}{2}, i_{2}+\frac{1}{2}, i_{3}+\frac{1}{2}}^{n}$, and the values of $\omega$ are obtained by averaging the values of $\omega$ computed from the solutions $\boldsymbol{U}$ at $t^{n}$, e.g.

$$
\omega_{i_{1}+1, i_{2}+\frac{1}{2}, i_{3}+\frac{1}{2}}:=\frac{1}{4}\left(\omega_{i_{1}+1, i_{2}+1, i_{3}+1}+\omega_{i_{1}+1, i_{2}+1, i_{3}}+\omega_{i_{1}+1, i_{2}, i_{3}+1}+\omega_{i_{1}+1, i_{2}, i_{3}}\right) .
$$

In our numerical tests, the total iteration number $\mu$ is taken as 10, unless otherwise stated.

Once the mesh $\left\{\boldsymbol{x}_{i_{1}+\frac{1}{2}, i_{2}+\frac{1}{2}, i_{3}+\frac{1}{2}}^{[\mu]}\right\}$ is obtained, the final adaptive mesh is given by

$$
\boldsymbol{x}_{i_{1}+\frac{1}{2}, i_{2}+\frac{1}{2}, i_{3}+\frac{1}{2}}^{n+1}:=\boldsymbol{x}_{i_{1}+\frac{1}{2}, i_{2}+\frac{1}{2}, i_{3}+\frac{1}{2}}^{n}+\Delta_{\tau}\left(\delta_{\tau} \boldsymbol{x}\right)_{i_{1}+\frac{1}{2}, i_{2}+\frac{1}{2}, i_{3}+\frac{1}{2}}^{n}
$$

where

$$
\left(\delta_{\tau} \boldsymbol{x}\right)_{i_{1}+\frac{1}{2}, i_{2}+\frac{1}{2}, i_{3}+\frac{1}{2}}^{n}:=\boldsymbol{x}_{i_{1}+\frac{1}{2}, i_{2}+\frac{1}{2}, i_{3}+\frac{1}{2}}^{[\mu]}-\boldsymbol{x}_{i_{1}+\frac{1}{2}, i_{2}+\frac{1}{2}, i_{3}+\frac{1}{2}}^{n}
$$


and the parameter $\Delta_{\tau}$ is used to limit the movement of mesh points

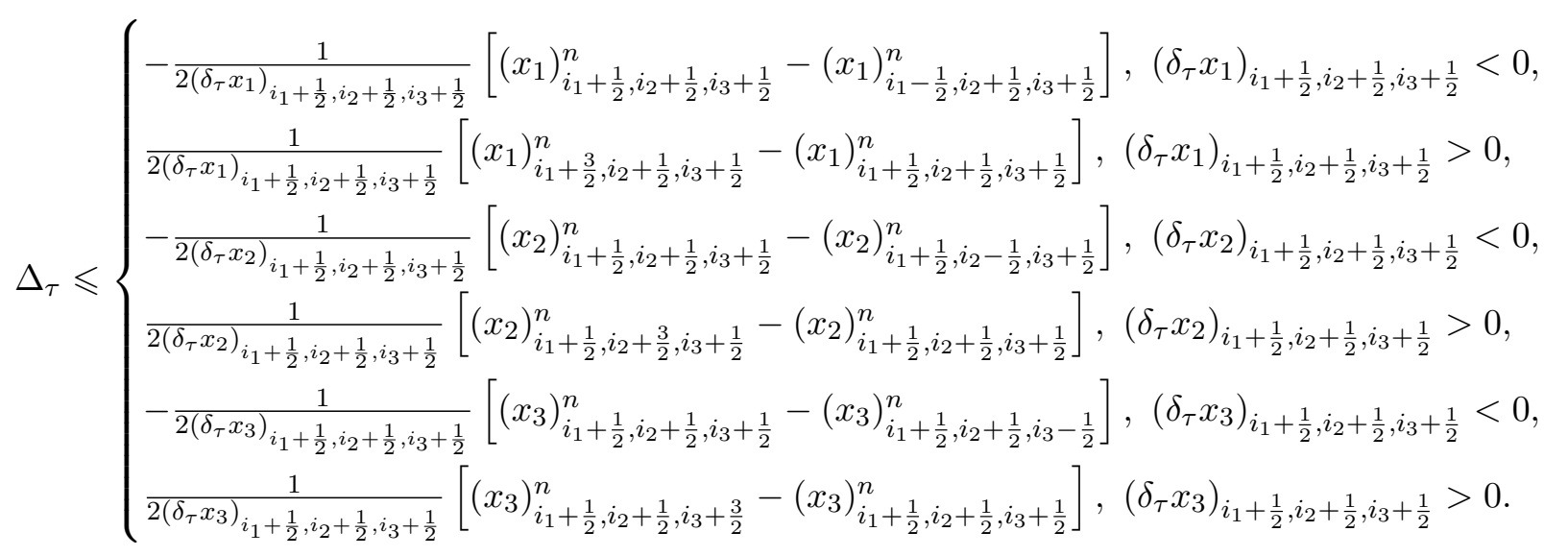

Finally, the mesh velocity at $t=t_{n}$ in (3.8) is defined by

$$
\dot{\boldsymbol{x}}_{i_{1}+\frac{1}{2}, i_{2}+\frac{1}{2}, i_{3}+\frac{1}{2}}^{n}:=\Delta_{\tau}\left(\delta_{\tau} \boldsymbol{x}\right)_{i_{1}+\frac{1}{2}, i_{2}+\frac{1}{2}, i_{3}+\frac{1}{2}}^{n} / \Delta t_{n}
$$

where $\Delta t_{n}$ is the time step size, determined by (5.1).

\section{Numerical results}

This section conducts several numerical experiments to validate the performance of our schemes. Our schemes are implemented in parallel by utilizing the MPI parts of the PLUTO code [41], and all simulations are performed with the CPU nodes of the High-performance Computing Platform of Peking University (Linux redhat environment, two Intel Xeon E5-2697A V4 (16 cores ×2) per node, and core frequency of $2.6 \mathrm{GHz}$ ). Unless otherwise stated, the adiabatic index $\Gamma$ is taken as $5 / 3$ and the time step size $\Delta t_{n}$ is determined by the usual CFL condition

$$
\Delta t_{n} \leqslant \frac{\mathrm{CFL}}{\sum_{k=1}^{3} \max _{\boldsymbol{i}} \varrho_{k, \boldsymbol{i}}^{n} / \Delta \xi_{k}}
$$

where $\varrho_{k, i}$ is the spectral radius of the eigen-matrix in the $i_{k}$-direction of $(3.1)$, and the CFL number is taken as 0.4 for $2 \mathrm{D}$ cases and 0.3 for $3 \mathrm{D}$ cases. Moreover, except for a comparison in Example 5.6, all numerical results are obtained by using VCL1 and SSP second-order RK method (still denoted VCL1 for the sake of brevity).

\section{1. $2 D$ results}

Example 5.1 (2D vortex problem). This 2D relativistic isentropic vortex problem, being a modification of the vortex problem in [33], is used to test the accuracy. It describes a relativistic vortex 
moves with a constant speed of magnitude $w$ in $(-1,-1)$ direction. Specially, the initial rest-mass density, pressure and velocities are given by

$$
\begin{aligned}
& \rho\left(x_{1}, x_{2}\right)=\left(1-C_{1} e^{1-r^{2}}\right)^{\frac{1}{\Gamma-1}}, \quad p=\rho^{\Gamma}, \\
& v_{1}=\frac{1}{1-w\left(\widetilde{v}_{1}+\widetilde{v}_{2}\right) / \sqrt{2}}\left[\frac{\widetilde{v}_{1}}{\gamma}-\frac{w}{\sqrt{2}}+\frac{\gamma w^{2}}{2(\gamma+1)}\left(\widetilde{v}_{1}+\widetilde{v}_{2}\right)\right], \\
& v_{2}=\frac{1}{1-w\left(\widetilde{v}_{1}+\widetilde{v}_{2}\right) / \sqrt{2}}\left[\frac{\widetilde{v}_{2}}{\gamma}-\frac{w}{\sqrt{2}}+\frac{\gamma w^{2}}{2(\gamma+1)}\left(\widetilde{v}_{1}+\widetilde{v}_{2}\right)\right],
\end{aligned}
$$

where

$$
\begin{aligned}
& C_{1}=\frac{(\Gamma-1) / \Gamma}{8 \pi^{2}} \epsilon^{2}, r=\sqrt{\widetilde{x}_{1}^{2}+\widetilde{x}_{2}^{2}}, \widetilde{x}_{1}=x_{1}+\frac{\gamma-1}{2}\left(x_{1}+x_{2}\right)-1, \\
& \widetilde{x}_{2}=x_{2}+\frac{\gamma-1}{2}\left(x_{1}+x_{2}\right)-1, \quad \gamma=\frac{1}{\sqrt{1-w^{2}}}, \\
& \left(\widetilde{v}_{1}, \widetilde{v}_{2}\right)=\left(-\widetilde{x}_{2}, \widetilde{x}_{1}\right) f, f=\sqrt{\frac{C_{2}}{1+C_{2} r^{2}}}, \quad C_{2}=\frac{2 \Gamma C_{1} e^{1-r^{2}}}{2 \Gamma-1-\Gamma C_{1} e^{1-r^{2}}} .
\end{aligned}
$$

The computational domain $\Omega_{c}$ and the parameters $w$ and $\epsilon$ are taken as $[-5,5] \times[-5,5]$ with periodic boundary conditions, $0.5 \sqrt{2}$, and 5 , respectively, the monitor function is chosen as (4.4) with $\alpha=20, \sigma=\rho$, and the number of the Jacobi iterations is 3 . Table 5.1 lists the errors in the rest-mass density $\rho$ and orders of convergence obtained by using our ES moving mesh scheme with $N \times N$ cells. It can be seen that the adaptive ES scheme can achieve second-order accuracy. Figure 5.1 plots the adaptive meshes of $N=40$ at different times, which show that the concentration of the mesh points well follows the propagation of the vortex. Figure 5.2 presents contour of $\rho$ with 40 equally spaced contour lines obtained by the adaptive ES scheme and the changes of the total entropy $\sum_{i_{1}, i_{2}} J_{i_{1}, i_{2}} \eta\left(\boldsymbol{U}_{i_{1}, i_{2}}\right) \Delta \xi_{1} \Delta \xi_{2}$ with respect to time obtained by the adaptive EC and ES schemes with $N=320$. We can see that the total entropy of the adaptive EC scheme almost keeps conservative, while the total entropy of the adaptive ES scheme decays as expected.

\begin{tabular}{r|cc|cc|cc}
\hline$N$ & $\ell^{1}$ error & order & $\ell^{2}$ error & order & $\ell^{\infty}$ error & order \\
\hline 20 & $1.371 \mathrm{e}-02$ & - & $3.947 \mathrm{e}-02$ & - & $2.360 \mathrm{e}-01$ & - \\
40 & $7.458 \mathrm{e}-03$ & 0.88 & $1.999 \mathrm{e}-02$ & 0.98 & $1.250 \mathrm{e}-01$ & 0.92 \\
80 & $2.385 \mathrm{e}-03$ & 1.64 & $6.934 \mathrm{e}-03$ & 1.53 & $5.217 \mathrm{e}-02$ & 1.26 \\
160 & $5.561 \mathrm{e}-04$ & 2.10 & $1.817 \mathrm{e}-03$ & 1.93 & $1.766 \mathrm{e}-02$ & 1.56 \\
320 & $1.251 \mathrm{e}-04$ & 2.15 & $4.449 \mathrm{e}-04$ & 2.03 & $4.723 \mathrm{e}-03$ & 1.90 \\
\hline
\end{tabular}

Table 5.1: Example 5.1: Errors and orders of convergence in $\rho$ at $t=4$. 


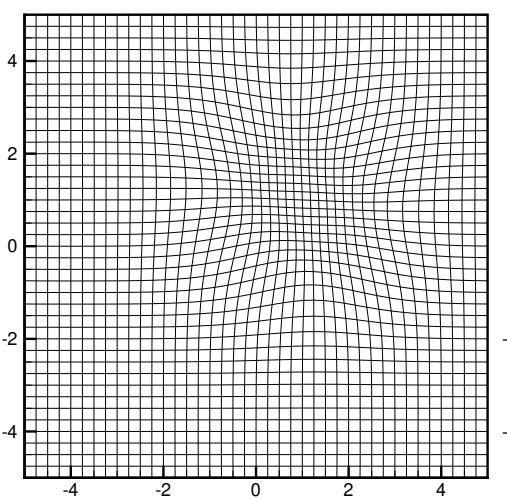

(a) $t=0$

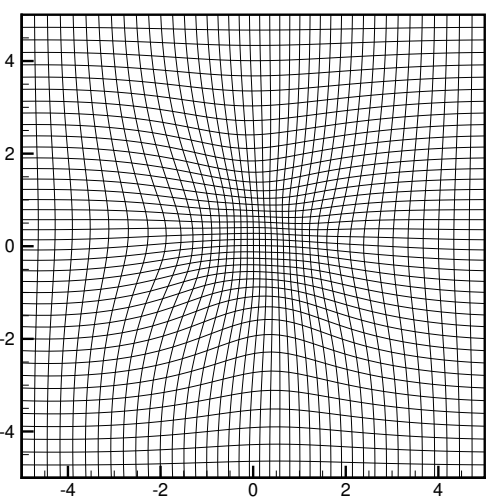

(b) $t=2$

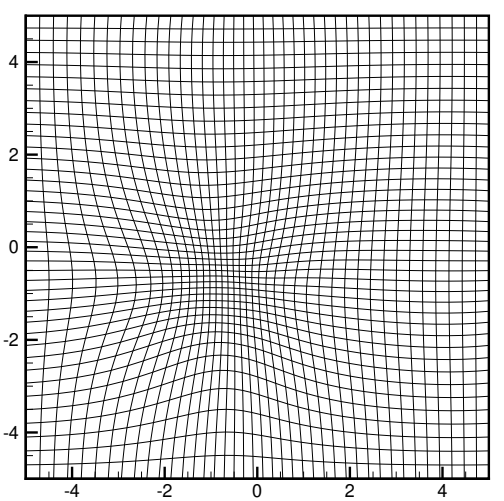

(c) $t=4$

Figure 5.1: Example 5.1: The adaptive meshes of $N=40$ at different times.
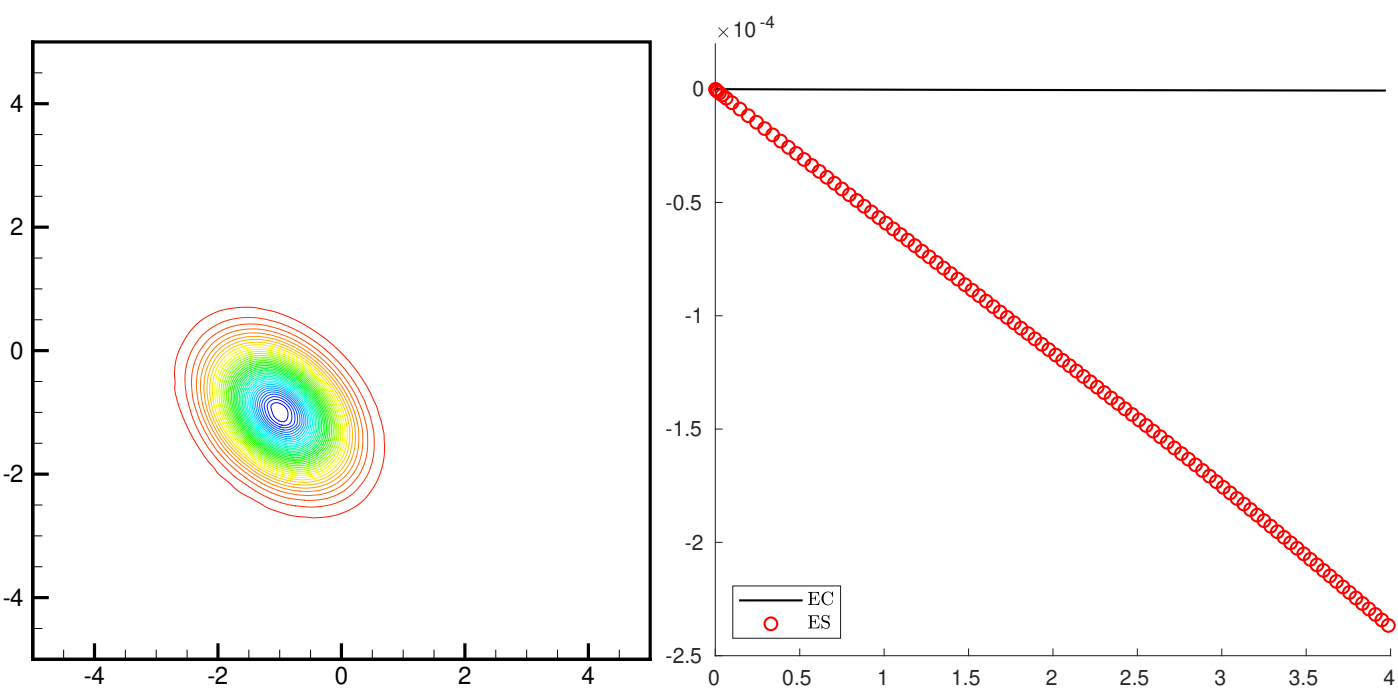

Figure 5.2: Example 5.1. Left: contour of $\rho$ with 40 equally spaced contour lines; right: change of the total entropy in $t . N=320$.

Example 5.2 (Riemann problem I). The initial data are

$$
\left(\rho, v_{1}, v_{2}, p\right)= \begin{cases}(0.5,0.5,-0.5,5), & x_{1}>0.5, x_{2}>0.5, \\ (1,0.5,0.5,5), & x_{1}<0.5, x_{2}>0.5, \\ (3,-0.5,0.5,5), & x_{1}<0.5, x_{2}<0.5, \\ (1.5,-0.5,-0.5,5), & x_{1}>0.5, x_{2}<0.5 .\end{cases}
$$

It will describe the interaction of four contact discontinuities (vortex sheets) with the same sign (the negative sign).

The monitor function is chosen as (4.4) with $\alpha=1200$ and $\sigma=\ln \rho$. Figure 5.3 shows the adaptive mesh, the contours of the density logarithms $\ln \rho$ with 40 equally spaced lines, and the 
cut of $\ln \rho$ along $x_{2}=x_{1}$ at $t=0.4$. It is seen that the four initial vortex sheets interact each other to form a spiral with the low rest-mass density around the center of the domain as time increases, which is the typical cavitation phenomenon in gas dynamics, and the adaptive mesh points well concentrate near the large gradient area of $\ln \rho$ as expected, which agrees well with the important features. Moreover, the solution on the adaptive moving mesh with $200 \times 200$ cells is better than that on the uniform mesh with the same cells, and is comparable to that on the uniform mesh with $600 \times 600$ cells according to the cut lines. Those verify the effectiveness of the present adaptive moving mesh strategy. The CPU times in Table 5.2 clearly highlight the efficiency of the adaptive moving mesh scheme, since it takes only $13.9 \%$ CPU time of the uniform mesh with $600 \times 600$ cells.

\begin{tabular}{l|ccc}
\hline & adaptive $(200 \times 200$ cells $)$ & uniform $(200 \times 200$ cells $)$ & uniform $(600 \times 600$ cells $)$ \\
\hline Example 5.2 & $1 \mathrm{~m} 08 \mathrm{~s}$ & $20 \mathrm{~s}$ & $7 \mathrm{~m} 47 \mathrm{~s}$ \\
Example 5.3 & $1 \mathrm{~m} 44 \mathrm{~s}$ & $18 \mathrm{~s}$ & $7 \mathrm{~m} 01 \mathrm{~s}$ \\
Example 5.4 & $2 \mathrm{~m} 48 \mathrm{~s}$ & $19 \mathrm{~s}$ & $7 \mathrm{~m} 16 \mathrm{~s}$ \\
\hline
\end{tabular}

Table 5.2: CPU times of Examples 5.2-5.4.
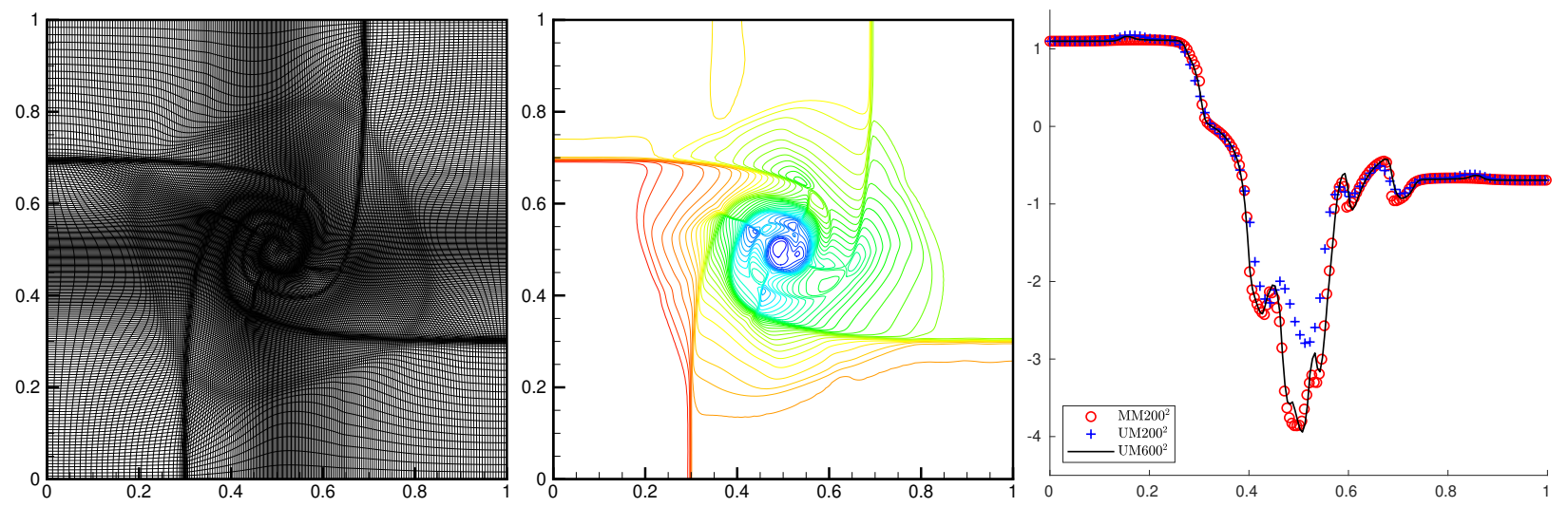

Figure 5.3: Example 5.2: From left to right: adaptive mesh of $200 \times 200$ cells, contour of $\ln \rho$ with 40 equally spaced contour lines, and $\ln \rho$ along $x_{2}=x_{1}$ at $t=0.4$.

Example 5.3 (Riemann problem II). The initial data are

$$
\left(\rho, v_{1}, v_{2}, p\right)= \begin{cases}(1,0,0,1), & x_{1}>0.5, x_{2}>0.5, \\ (0.5771,-0.3529,0,0.4), & x_{1}<0.5, x_{2}>0.5, \\ (1,-0.3529,-0.3529,1), & x_{1}<0.5, x_{2}<0.5, \\ (0.5771,0,-0.3529,0.4), & x_{1}>0.5, x_{2}<0.5,\end{cases}
$$

which is about the interaction of four rarefaction waves. 
The monitor function is the same as that in the last example. Figure 5.4 shows the adaptive mesh, the contours of the density logarithms $\ln \rho$ with 40 equally spaced lines, and $\ln \rho$ along $x_{2}=x_{1}$ at $t=0.4$. The CPU times are listed in Table 5.2. The results show that those four initial discontinuities first evolve as four rarefaction waves and then interact each other and form two (almost parallel) curved shock waves perpendicular to the line $x_{2}=x_{1}$ as time increases. It is seen that the adaptive mesh method effectively captures the important features such as rarefaction waves and shock waves, and is well comparable to the fixed mesh method with a finer mesh.
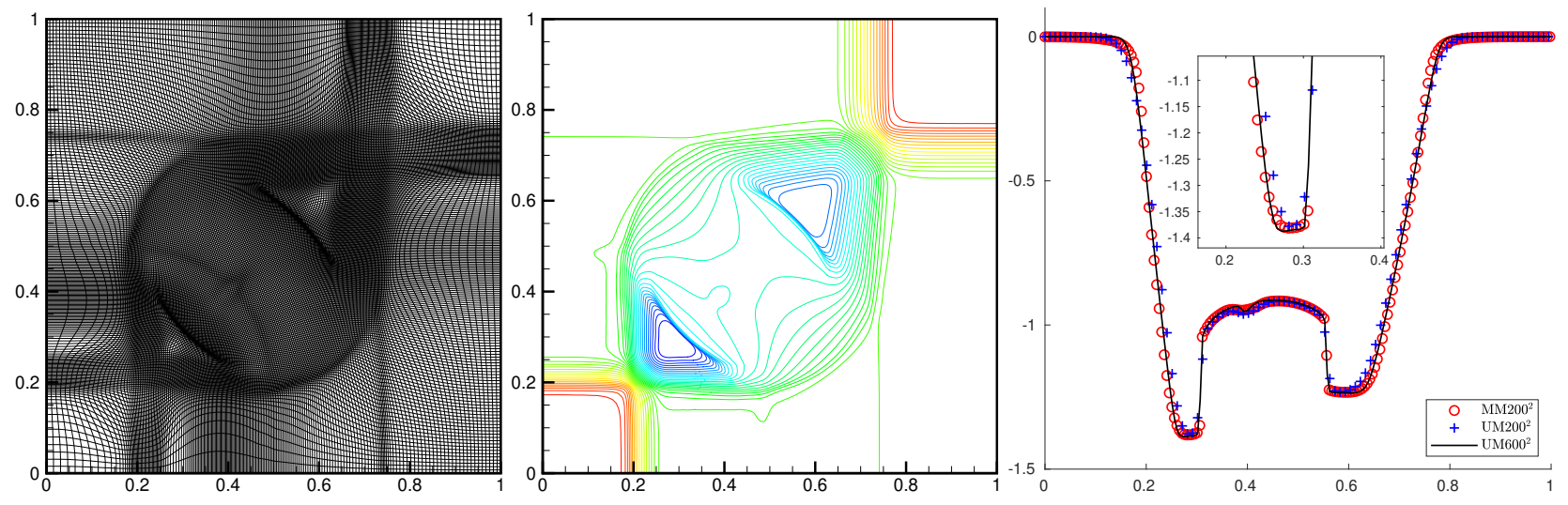

Figure 5.4: Example 5.3: From left to right: adaptive mesh of $200 \times 200$ cells, contour of $\ln \rho$ with 40 equally spaced contour lines, and $\ln \rho$ along $x_{2}=x_{1}$ at $t=0.4$.

Example 5.4 (Riemann problem III). The initial data are

$$
\left(\rho, v_{1}, v_{2}, p\right)= \begin{cases}(0.035145216124503,0,0,0.162931056509027), & x_{1}>0.5, x_{2}>0.5, \\ (0.1,0.7,0,1), & x_{1}<0.5, x_{2}>0.5, \\ (0.5,0,0,1), & x_{1}<0.5, x_{2}<0.5, \\ (0.1,0,0.7,1), & x_{1}>0.5, x_{2}<0.5,\end{cases}
$$

where the left and bottom discontinuities are two contact discontinuities and the top and right are two shock waves.

The monitor function is the same as above. The adaptive mesh, the contours of the density logarithms $\ln \rho$ with 40 equally spaced lines, and $\ln \rho$ cut along $x_{2}=x_{1}$ at $t=0.4$ are present in Figure 5.5. The initial discontinuities interact each other and form a "mushroom cloud" around the point $(0.5,0.5)$, which is well captured by the adaptive moving mesh method with the chosen monitor function. Similar to the last two examples, the solution obtained by the adaptive moving mesh with $200 \times 200$ cells is much better than that on the same uniform cells, and agrees well with that with $600 \times 600$ uniform cells, while the adaptive moving mesh scheme only takes $34.6 \% \mathrm{CPU}$ time, see Table 5.2, showing the high efficiency of the adaptive scheme. 

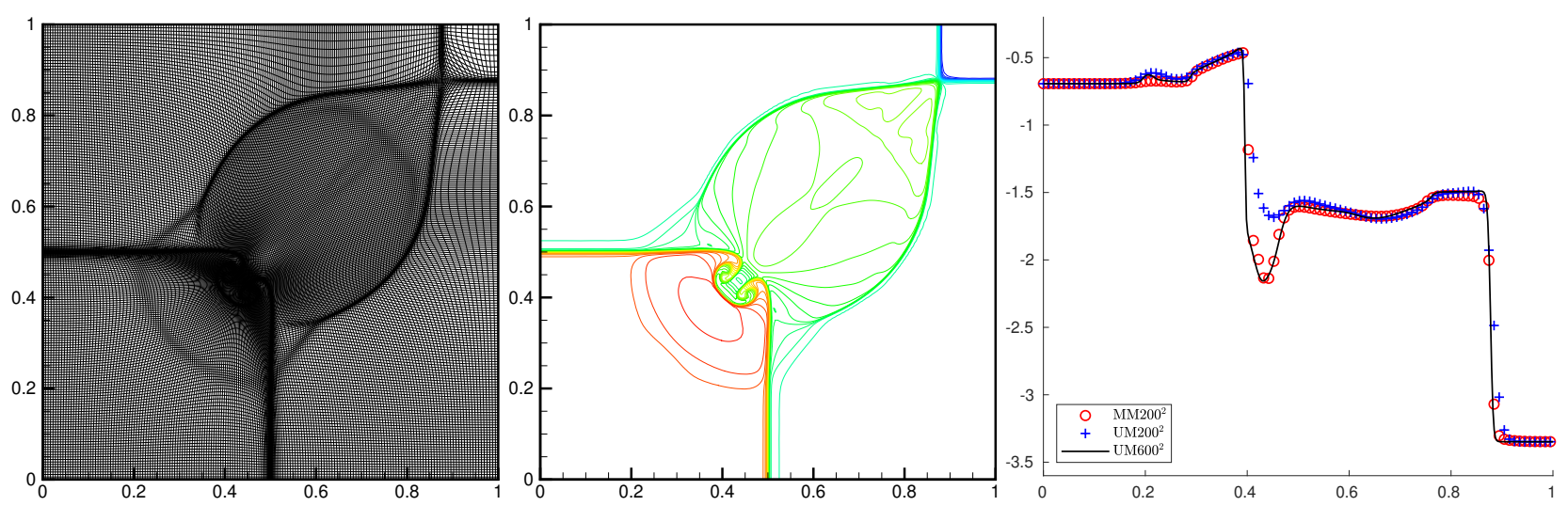

Figure 5.5: Example 5.4: From left to right: adaptive mesh of $200 \times 200$ cells, contour of $\ln \rho$ with 40 equally spaced contour lines, and $\ln \rho$ along $x_{2}=x_{1}$ at $t=0.4$.

\section{2. $3 D$ results}

Example 5.5 (3D smooth sine wave). This test is used to verify the accuracy of the 3D ES moving mesh scheme. The physical domain is a unit cube with periodic boundary conditions, and partitioned into $N \times N \times N$ cells. The exact solutions are given by

$$
\left(\rho, v_{1}, v_{2}, v_{3}, p\right)\left(x_{1}, x_{2}, x_{3}, t\right)=\left(1+0.2 \sin \left[2 \pi\left(x_{1}+x_{2}+x_{3}-\left(v_{1}+v_{2}+v_{3}\right) t\right)\right], 0.2,0.4,0.6,1\right) .
$$

The monitor function and the number of the Jacobi iteration are the same as those in the 2D accuracy test. Table 5.3 lists the errors and the orders of convergence in $\rho$ at $t=0.1$. Figure 5.6 displays the adaptive mesh at the final time. The conclusions are similar to the $2 \mathrm{D}$ case.

\begin{tabular}{r|cc|cc|cc}
\hline$N$ & $\ell^{1}$ error & order & $\ell^{2}$ error & order & $\ell^{\infty}$ error & order \\
\hline 20 & $2.085 \mathrm{e}-02$ & - & $2.551 \mathrm{e}-02$ & - & $4.699 \mathrm{e}-02$ & - \\
40 & $1.173 \mathrm{e}-02$ & 0.83 & $1.446 \mathrm{e}-02$ & 0.82 & $2.638 \mathrm{e}-02$ & 0.83 \\
80 & $4.166 \mathrm{e}-03$ & 1.49 & $6.266 \mathrm{e}-03$ & 1.21 & $1.455 \mathrm{e}-02$ & 0.86 \\
160 & $1.287 \mathrm{e}-03$ & 1.69 & $2.239 \mathrm{e}-03$ & 1.48 & $6.524 \mathrm{e}-03$ & 1.16 \\
320 & $3.319 \mathrm{e}-04$ & 1.96 & $5.992 \mathrm{e}-04$ & 1.90 & $2.025 \mathrm{e}-03$ & 1.69 \\
\hline
\end{tabular}

Table 5.3: Example 5.5: Errors and orders of convergence in $\rho$ at $t=0.1$.

Example 5.6 (Spherical symmetric Riemann problem). To examine the performance of the 3D scheme, we first consider this Riemann problem with a reference solution obtained by using a second-order TVD scheme to solve the RHD equations in $1 \mathrm{D}$ spherical coordinates. The initial data are

$$
\left(\rho, v_{1}, v_{2}, v_{3}, p\right)= \begin{cases}(10,0,0,0,40 / 3), & r=\sqrt{x_{1}^{2}+x_{2}^{2}+x_{3}^{2}}<0.5 \\ \left(1,0,0,0,10^{-6}\right), & \text { otherwise }\end{cases}
$$




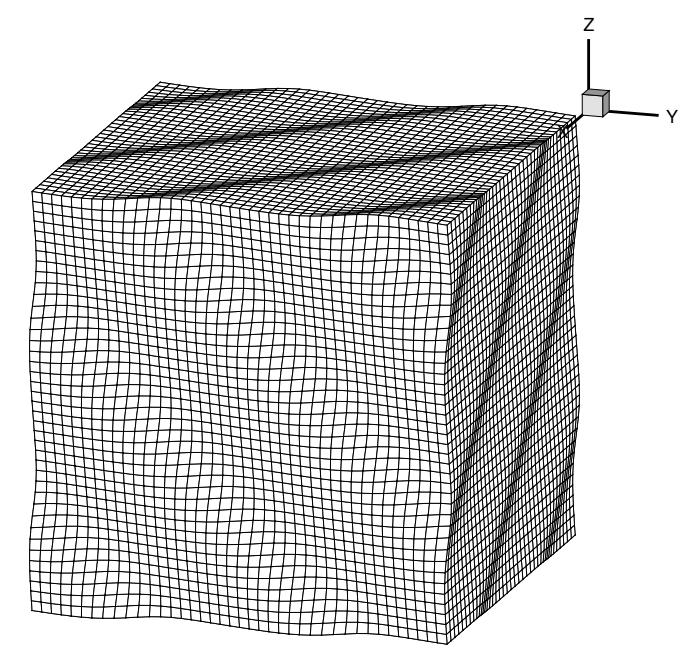

Figure 5.6: Example 5.5: Adaptive mesh of $40 \times 40 \times 40$ cells at $t=0.1$.

The monitor function is chosen as (4.4) with $\alpha=1000$ and $\sigma=\ln \rho$. Figures 5.7 and 5.8 give the adaptive mesh and the comparison of the density $\rho$ and the magnitude of velocity $|\boldsymbol{v}|$ along the line connecting $(0,0,0)$ and $(1,1,1)$ at $t=0.4$ obtained by using $100 \times 100 \times 100$ or $200 \times 200 \times 200$ cells, respectively. It is obvious that all the schemes give correct solutions, while the adaptive moving mesh scheme gives better results than the uniform mesh even with double mesh cells in each direction, since the adaptive mesh concentrates near where large gradient in $\ln \rho$ occurs, increasing the resolution near the discontinuities. From Table 5.4, the CPU time of the ES moving mesh scheme is $18.0 \%$ of the refined uniform mesh, showing the high efficiency of the adaptive moving mesh scheme.

The performances of VCL1 and VCL2 are compared in Figure 5.9. The left figure shows the evolution of the logarithm of the difference (in the $\ell^{1}$-norm) between the Jacobian $\left\{J_{i}^{n}\right\}$ updated by VCL1 or VCL2 and $\left\{\tilde{J}_{i}^{n}\right\}$ obtained by the direct discretization of the first equation in (3.9). It can be seen that using VCL1 with the SSP second- and third-order RK methods (abbreviated respectively as RK2 and RK3) gives almost the same error, which is larger (about two order of magnitude) than VCL2 with RK2. The error obtained by VCL2 with RK3 is nearly $10^{-12}$, verifying the analysis in Section 3.2. The right figure presents a comparison of the rest-mass density $\rho$, where no obvious difference is observed. The CPU time of the adaptive ES scheme with VCL2 is about $4 \%$ (resp.6\%) larger than that of the adaptive ES scheme with VCL1 when RK2 (resp. RK3) is used. In view of those, VCL1 is used in all other examples.

Example 5.7 (Shock-bubble interaction problem). This example considers a moving planar shock interacts with a light bubble within the domain $[0,325] \times[-45,45] \times[-45,45]$. The detailed setup 


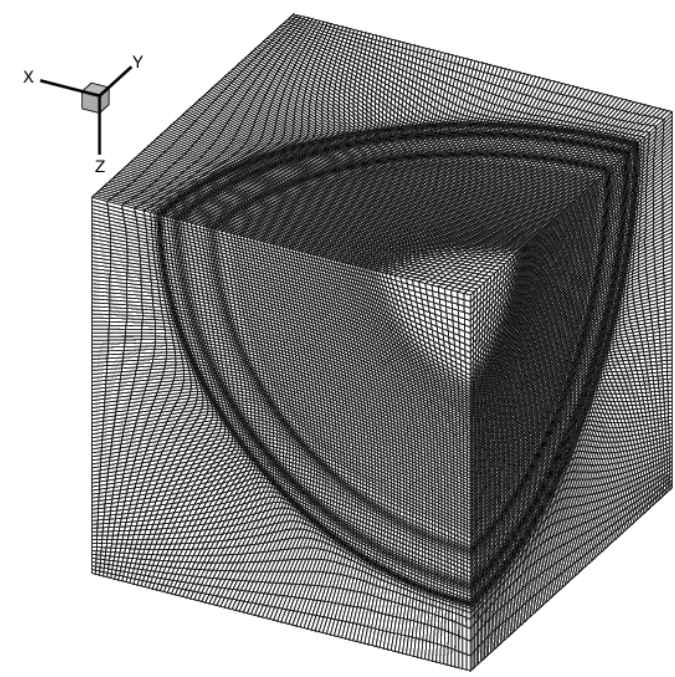

Figure 5.7: Example 5.6: Adaptive mesh at $t=0.4$.

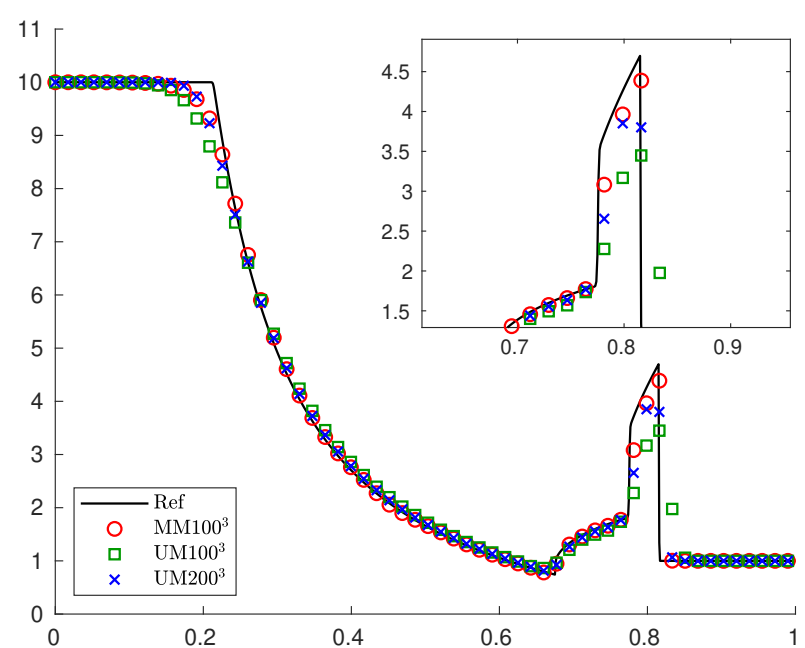

(a) $\rho$

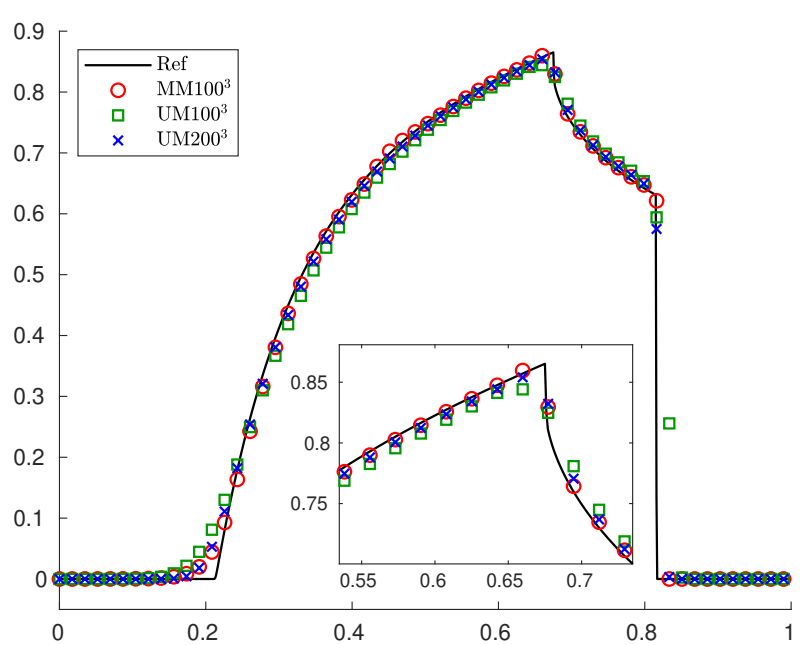

(b) $|\boldsymbol{v}|$

Figure 5.8: Example 5.6: $\rho$ and $|\boldsymbol{v}|$ along the line connecting $(0,0,0)$ and $(1,1,1)$ at $t=0.4$.

\begin{tabular}{l|ccc}
\hline & adaptive mesh (cells) & uniform mesh (cells) & fine uniform (cells) \\
\hline Example 5.6 & $2 \mathrm{~m} 48 \mathrm{~s}(100 \times 100 \times 100)$ & $1 \mathrm{~m} 05 \mathrm{~s}(100 \times 100 \times 100)$ & $15 \mathrm{~m} 32 \mathrm{~s}(200 \times 200 \times 200)$ \\
Example 5.7 & $1 \mathrm{~h} 24 \mathrm{~m} 20 \mathrm{~s}(325 \times 90 \times 90)$ & $26 \mathrm{~m} 07 \mathrm{~s}(325 \times 90 \times 90)$ & $6 \mathrm{~h} 27 \mathrm{~m} 31 \mathrm{~s}(650 \times 180 \times 180)$ \\
\hline
\end{tabular}

Table 5.4: CPU times of Examples 5.6-5.7 (32 cores are used).

can be found in [24]. The initial pre- and post-shock states are

$$
\left(\rho, v_{1}, v_{2}, v_{3}, p\right)= \begin{cases}(1,0,0,0,0.05), & x_{1}<265, \\ (1.865225080631180,-0.196781107378299,0,0,0.15), & x_{1}>265,\end{cases}
$$




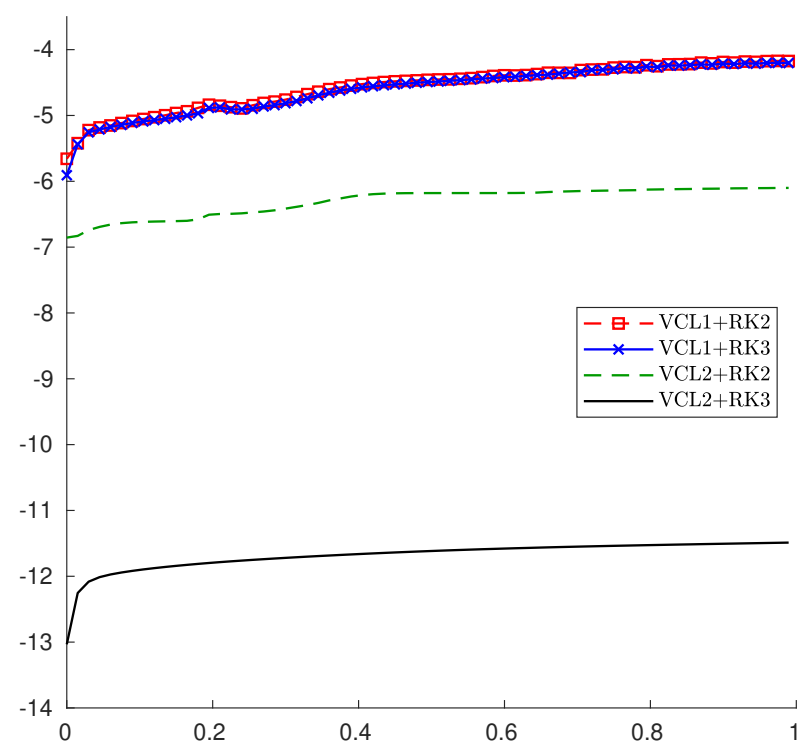

(a) $\log _{10}\left(\left\|J^{n}-\tilde{J}^{n}\right\|_{\ell^{1}}\right)$

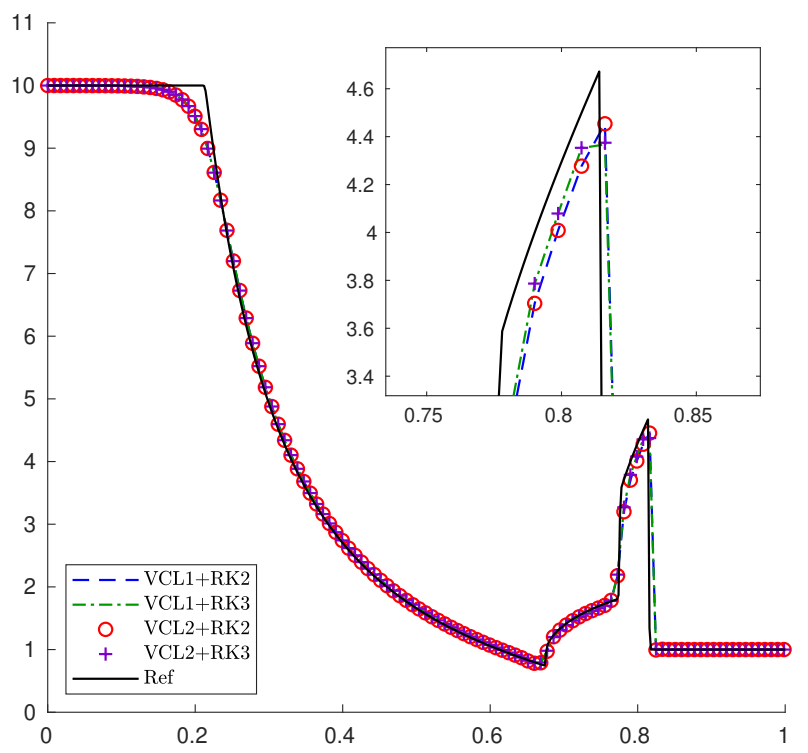

(b) $\rho$

Figure 5.9: A comparison of VCL1 and VCL2 with RK2 or RK3. The Jacobian $\left\{J_{i}^{n}\right\}$ is updated by VCL1 or VCL2, while $\left\{\tilde{J}_{i}^{n}\right\}$ is obtained by the direct discretization of the first equation in (3.9).

and the state of the bubble is

$$
\left(\rho, v_{1}, v_{2}, v_{3}, p\right)=(0.1358,0,0,0,0.05), \quad \sqrt{\left(x_{1}-215\right)^{2}+x_{2}^{2}+x_{3}^{2}} \leqslant 25 .
$$

The monitor is the same as that in the last example. Figure 5.10 shows close-up of the adaptive mesh and the 6 iso-surfaces of $\rho$ equally spaced from 0.55 to 1.75, and two surface meshes near the bubble at $t=450$. It is seen that the adaptive mesh points well concentrate near the planar shock and the bubble according to the monitor function. Figure 5.11 presents the schlieren images on the slice $x_{2}=0$ of the rest-mass density $\rho$ at $t=90,180,270,360,450$ (from top to bottom) with $325 \times 90 \times 90$ moving mesh, $325 \times 90 \times 90$ uniform mesh and $650 \times 180 \times 180$ moving mesh (from left ro tight), respectively. Those plots clearly show the dynamics of the interaction between the shock wave and the bubble, and the sharp interfaces of the bubble at different output times are well captured by the moving mesh scheme. The ES adaptive moving mesh scheme only takes 21.7\% CPU time of the refined uniform mesh from Table 5.4, and gives better results, highlighting its high efficiency.

\section{Conclusion}

This paper presented the ES adaptive moving mesh schemes for the $2 \mathrm{D}$ and $3 \mathrm{D}$ special RHD equations, which could be viewed as an extension of the second-order ES schemes in [14] to the adaptive moving mesh. Our schemes were built on the ES finite volume approximation of the RHD 

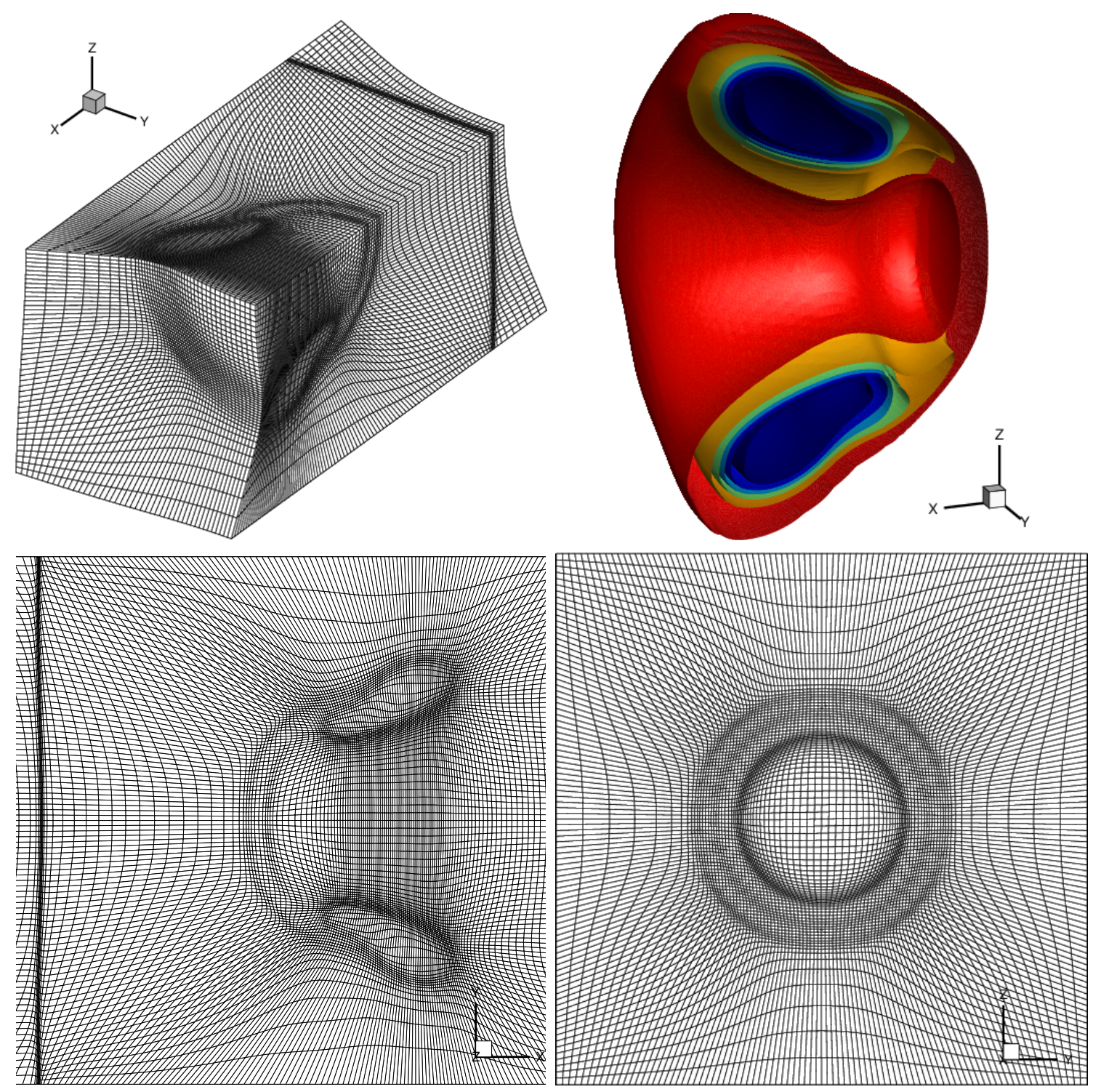

Figure 5.10: Example 5.7: Adaptive meshes and $\rho$ at $t=450$. Top left: close-up of the adaptive mesh, $i_{1} \in$ $[30,140], i_{2} \in[0,45], i_{3} \in[0,45]$; top right: 6 iso-surfaces of $\rho$; bottom left: the surface mesh with $i_{3}=i_{3,46+\frac{1}{2}}$; bottom right: the surface mesh on $i_{1}=i_{1,80+\frac{1}{2}}$.

equations in curvilinear coordinates, the discrete geometric conservation laws, and the adaptive mesh redistribution. Following the procedure in [13], we constructed the EC fluxes in curvilinear coordinates for the given entropy pair. To do that, a sufficient condition for the so-called two-point EC fluxes was first given. Its proof mimicked the derivation of the continuous entropy identity in curvilinear coordinates and utilized the discrete GCLs achieved by the conservative metrics method [53]. In order to avoid the numerical oscillation produced by the EC scheme around the discontinuities, some suitable dissipation term utilizing linear reconstruction with the minmod limiter in the scaled entropy variables was added to the EC flux to get the second-order accurate ES 

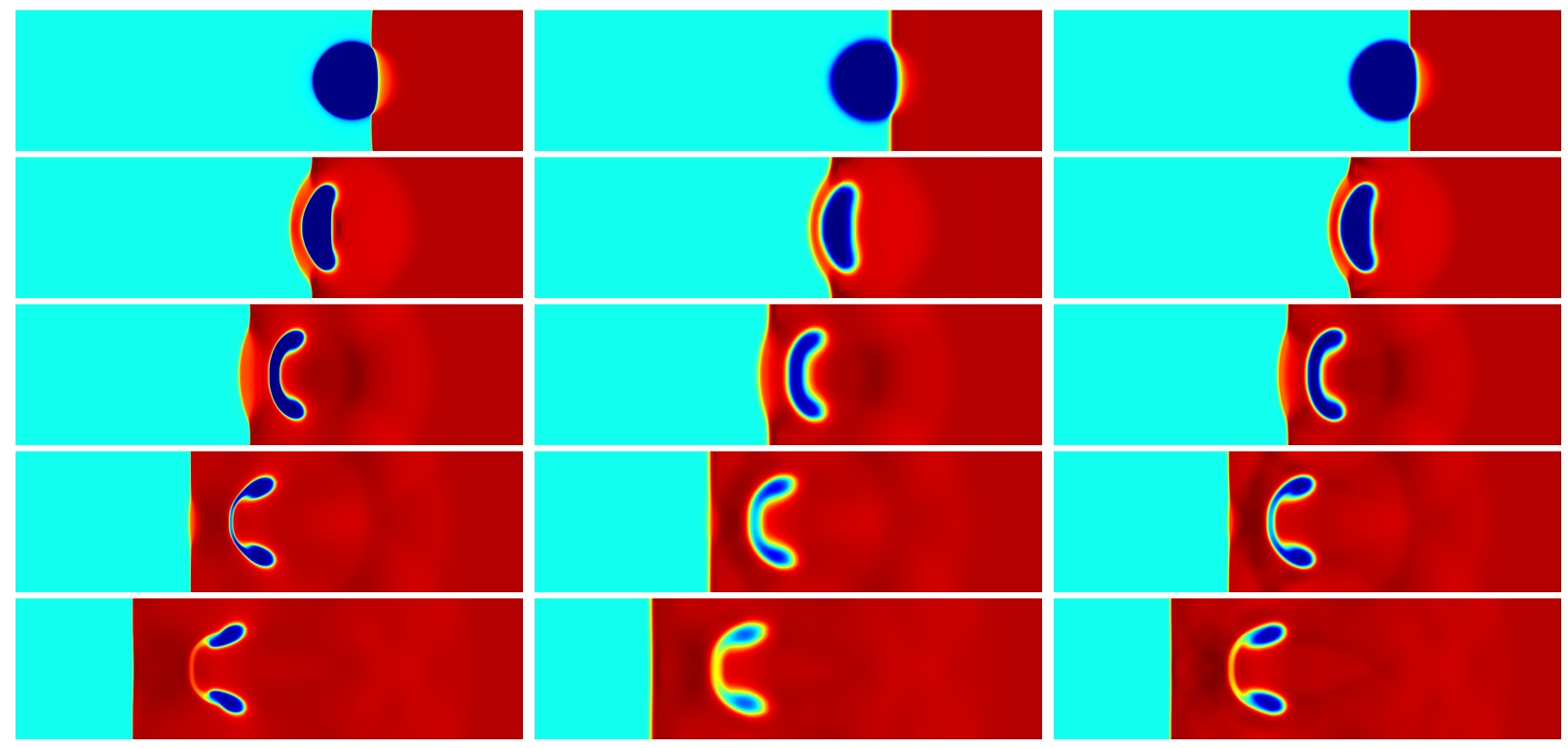

Figure 5.11: Example 5.7: $\rho$ on the slice $x_{2}=0$ at $t=90,180,270,360,450$ (from top to bottom). Left: moving mesh of $325 \times 90 \times 90$, middle: uniform mesh of $325 \times 90 \times 90$, right: uniform mesh of $650 \times 180 \times 180$.

scheme satisfying the semi-discrete entropy inequality. The fully discrete schemes were derived by integrating the above semi-discrete ES schemes in time by using the second-order accurate explicit strong-stability preserving Runge-Kutta schemes. The resulting fully-discrete scheme was proved to preserve the free-stream states and two approximations of the volume conservation law were given and compared. The first was easy to be implemented, while the second could well approach to the value of the Jacobian $J$ calculated by its definition, i.e. the first equation of (3.9). The mesh points were adaptively moved or redistributed by solving the Euler-Lagrange equation of the mesh adaption functional on the computational mesh at each time step with suitably chosen monitor functions. Several 2D and 3D numerical results showed that the ES adaptive moving mesh schemes effectively captured the localized structures, such as sharp transitions or discontinuities, and were more efficient than their counterparts on uniform mesh.

\section{Acknowledgments}

The authors were partially supported by the Special Project on High-performance Computing under the National Key R\&D Program (No. 2016YFB0200603), Science Challenge Project (No. TZ2016002), the Sino-German Cooperation Group Project (No. GZ 1465), the National Natural Science Foundation of China (No. 11421101), and High-performance Computing Platform of Peking University. 


\section{References}

[1] Y. Abe, N. Iizuka, T. Nonomura, and K. Fujii, Conservative metric evaluation for high-order finite difference schemes with the GCL identities on moving and deforming grids, J. Comput. Phys., 232 (2013), 14-21.

[2] T.J. Barth, Numerical methods for gasdynamic systems on unstructured meshes, in D. Kroner, M. Ohlberger, and C. Rohde, editors, An Introduction to Recent Developments in Theory and Numerics for Conservation Laws, Springer (1999), 195-285.

[3] D. Bhoriya and H. Kumar, Entropy-stable schemes for relativistic hydrodynamics equations, Z. Angew. Math. Phys., 71 (2020), 1-29.

[4] J.U. Brackbill, An adaptive grid with directional control, J. Comput. Phys., 108 (1993), 38-50.

[5] J.U. Brackbill and J.S. Saltzman, Adaptive zoning for singular problems in two dimensions, J. Comput. Phys., 46 (1982), 342-368.

[6] C.J. Budd, W.Z. Huang, and R.D. Russell, Adaptivity with moving grids, Acta Numer., 18 (2009), 111-241.

[7] W.M. Cao, W.Z. Huang, and R.D. Russell, A study of monitor functions for two-dimensional adaptive mesh generation, SIAM J. Sci. Comput., 20 (1999), 1978-1994.

[8] M.H. Carpenter, T.C. Fisher, E.J. Nielsen, and S.H. Frankel, Entropy stable spectral collocation schemes for the Navier-Stokes equations: Discontinuous interfaces, SIAM J. Sci. Comput., 36 (2014), B835-B867.

[9] P. Chandrashekar and C. Klingenberg, Entropy stable finite volume scheme for ideal compressible MHD on 2D Cartesian meshes, SIAM J. Numer. Anal., 54 (2016), 1313-1340.

[10] T.H. Chen and C.W. Shu, Review of entropy stable discontinuous Galerkin methods for systems of conservation laws on unstructured simplex meshes, CSIAM Trans. Appl. Math., 1 (2020), $1-52$.

[11] L. Del Zanna, N. Bucciantini, and P. Londrillo, An efficient shock-capturing central-type scheme for multi-dimensional relativistic flows, I: Hydrodynamics, Astron. Astrophys., 3 (2002), 1177-1186.

[12] A. Dolezal and S.S.M. Wong, Relativistic hydrodynamics and essentially non-oscillatory shock capturing schemes, J. Comput. Phys., 120 (1995), 266-277. 
[13] J.M. Duan and H.Z. Tang, High-order accurate entropy stable nodal discontinuous Galerkin schemes for the ideal special relativistic magnetohydrodynamics, accepted by J. Comput. Phys., July 20, 2020. https://doi.org/10.1016/j.jcp.2020.109731.

[14] J.M. Duan and H.Z. Tang, High-order accurate entropy stable finite difference schemes for oneand two-dimensional special relativistic hydrodynamics, Adv. Appl. Math. Mech., 12 (2020), $1-29$.

[15] J.M. Duan and H.Z. Tang, High-order accurate entropy stable finite difference schemes for the shallow water magnetohydrodynamics, arXiv:2003.10081v2, (2020).

[16] F. Eulderink and G. Mellema, General relativistic hydrodynamics with a Roe solver, Astron. Astrophys. Suppl. Ser., 110 (1994), 34.

[17] T.C. Fisher and M.H. Carpenter, High-order entropy stable finite difference schemes for nonlinear conservation laws: Finite domains, J. Comput. Phys., 252 (2013), 518-557.

[18] U.S. Fjordholm, S. Mishra, and E. Tadmor, Well-balanced and energy stable schemes for the shallow water equations with discontinuous topography, J. Comput. Phys., 230 (2011), $5587-5609$.

[19] U.S. Fjordholm, S. Mishra, and E. Tadmor, Arbitrarily high-order accurate entropy stable essentially non-oscillatory schemes for systems of conservation laws, SIAM J. Numer. Anal., 50 (2012), 544-573.

[20] J.A. Font, Numerical hydrodynamics and magnetohydrodynamics in general relativity, Living Rev. Relativ., 11 (2008), 7.

[21] G.J. Gassner, A skew-symmetric discontinuous Galerkin spectral element discretization and its relation to SBP-SAT finite difference methods, SIAM J. Sci. Comput., 35 (2013), 1233-1253.

[22] S. Gottlieb, C.W. Shu, and E. Tadmor, Strong stability-preserving high-order time discretization methods, SIAM Review, 43 (2001), 89-112.

[23] J.Q. Han and H.Z. Tang, An adaptive moving mesh method for two-dimensional ideal magnetohydrodynamics, J. Comput. Phys., 220 (2007), 791-812.

[24] P. He and H.Z. Tang, An adaptive moving mesh method for two-dimensional relativistic hydrodynamics, Commun. Comput. Phys., 11 (2012), 114-146.

[25] P. He and H.Z. Tang, An adaptive moving mesh method for two-dimensional relativistic magnetohydrodynamics, Comput. Fluids, 60 (2012), 1-20. 
[26] A. Hiltebrand and S. Mishra, Entropy stable shock capturing space-time discontinuous Galerkin schemes for systems of conservation laws, Numer. Math., 126 (2014), 103-151.

[27] W.Z. Huang, Variational mesh adaptation: Isotropy and equidistribution, J. Comput. Phys., 174 (2001), 903-924.

[28] W.Z. Huang and R.D. Russell, Adaptive Moving Mesh Methods, Springer New York (2011).

[29] F. Ismail and P.L. Roe, Affordable, entropy-consistent Euler flux functions II : Entropy production at shocks, J. Comput. Phys., 228 (2009), 5410-5436.

[30] G.S. Jiang and C.W. Shu, On a cell enropy inequality for discontinuous Galerkin methods, Math. Comp., 62 (1994), 531-538.

[31] P.G. LeFloch, J.M. Mercier, and C. Rohde, Fully discrete entropy conservative schemes of arbitraty order, SIAM J. Numer. Anal., 40 (2002), 1968-1992.

[32] R. Li, T. Tao, and P.W. Zhang, Moving mesh methods in multiple dimensions based on harmonic maps, J. Comput. Phys., 170 (2001), 562-588.

[33] D. Ling, J.M. Duan, and H.Z. Tang, Physical-constraints-preserving Lagrangian finite volume schemes for one- and two-dimensional special relativistic hydrodynamics, J. Comput. Phys., 396 (2019), 507-543.

[34] J.M. Martí and E. Müller, Extension of the piecewise parabolic method to one-dimensional relativistic hydrodynamics, J. Comput. Phys., 123 (1996), 1-14.

[35] J.M. Martí and E. Müller, Numerical hydrodynamics in special relativity, Living Rev. Relativ., $6(2003), 7$.

[36] J.M. Martí and E. Müller, Grid-based methods in relativistic hydrodynamics and magnetohydrodynamics, Living Rev. Relativ., 1 (2015), 3.

[37] M.M. May and R.H. White, Hydrodynamic calculations of general-relativistic collapse, Phys. Rev., 141 (1966), 1232-1241.

[38] M.M. May and R.H. White, Stellar dynamics and gravitational collapse, in Methods Comput. Phys., Vol. 7, edited by B. Alder, S. Fernbach, and M. Rotenberg, New York: Academic (1967), 219-258.

[39] M.L. Merriam, An entropy-based approach to nonlinear stability, NASA-TM-101086 (1989). 
[40] A. Mignone and G. Bodo, An HLLC Riemman solver for relativistic flows-I Hydrodynamics, Mon. Not. R. Astron. Soc., 136 (2005), 126-136.

[41] A. Mignone, G. Bodo, S. Massaglia, T. Matsakos, O. Tesileanu, C. Zanni, and A. Ferrari, PLUTO: A numerical code for computational astrophysics, Astrophys. J. Suppl. Ser., 170 (2007), 228-242.

[42] A. Mignone, T. Plewa, and G. Bodo, The piecewise parabolic method for multidimensional relativistic fluid dynamics, Astron. Astrophys. Suppl. Ser., 160 (2005), 199-219.

[43] H.S. Pathak and R.K. Shukla, Adaptive finite-volume WENO schemes on dynamically redistributed grids for compressible Euler equations, J. Comput. Phys., 319 (2016), 200-230.

[44] W.Q. Ren and X.P. Wang, An iterative grid redistribution method for singular problems in multiple dimensions, J. Comput. Phys., 159 (2000), 246-273.

[45] V. Schneider, U. Katscher, D.H. Rischke, B. Waldhauser, J.A. Maruhn, and C.D. Munz, New algorithms for ultra-relativistic numerical hydrodynamics, J. Comput. Phys., 105 (1993), 92107.

[46] E. Tadmor, The numerical viscosity of entropy stable schemes for systems of conservation laws, I, Math. Comp., 49 (1987), 91-103.

[47] E. Tadmor, Entropy stability theory for difference approximations of nonlinear conservation laws and related time-dependent problems, Acta Numer., 12 (2003), 451-512.

[48] H.Z. Tang, A moving mesh method for the Euler flow calculations using a directional monitor function, Commun. Comput. Phys., 1 (2006), 656-676.

[49] H.Z. Tang and T. Tang, Adaptive mesh methods for one- and two-dimensional hyperbolic conservation laws, SIAM J. Numer. Anal., 41 (2003), 487-515.

[50] H.Z. Tang, T. Tang, and P.W. Zhang, An adaptive mesh redistribution method for nonlinear Hamilton-Jacobi equations in two- and three-dimensions, J. Comput. Phys., 188 (2003), 543572 .

[51] T. Tang, Moving mesh methods for computational fluid dynamics, Contemp. Math., 383 (2005), $141-173$.

[52] A. Tchekhovskoy, J.C. McKinney, and R. Narayan, WHAM: a WENO-based general relativistic numerical scheme - I. Hydrodynamics, Mon. Not. R. Astron. Soc., 379 (2007), 469-497. 
[53] P.D. Thomas and C.K. Lombard, Geometric conservation law and its application to flow computations on moving grids, AIAA J., 17 (1979), 1030-1037.

[54] D.S. Wang and X.P. Wang, A three-dimensional adaptive method based on the iterative grid redistribution, J. Comput. Phys., 199 (2004), 423-436.

[55] J.R. Wilson, Numerical study of fluid flow in a kerr space, Astrophys. J., 173 (1972), 431-438.

[56] A.M. Winslow, Numerical solution of the quasilinear Poisson equation in a nonuniform triangle mesh, J. Comput. Phys., 1 (1967), 149-172.

[57] A.R. Winters and G.J. Gassner, Affordable, entropy conserving and entropy stable flux functions for the ideal MHD equations, J. Comput. Phys., 304 (2016), 72-108.

[58] A.R. Winters and G.J. Gassner, An entropy stable finite volume scheme for the equations of shallow water magnetohydrodynamics, J. Sci. Comput., 67 (2016), 514-539.

[59] K.L. Wu and C.W. Shu, Entropy symmetrization and high-order accurate entropy stable numerical schemes for relativistic MHD equations, accepted by SIAM J. Sci. Comput., (2020).

[60] K.L. Wu and H.Z. Tang, High-order accurate physical-constraints-preserving finite difference WENO schemes for special relativistic hydrodynamics, J. Comput. Phys., 298 (2015), 539-564.

[61] K.L. Wu and H.Z. Tang, A direct Eulerian GRP scheme for spherically symmetric general relativistic hydrodynamics, SIAM J. Sci. Comput., 38 (2016), B458-B489.

[62] K.L. Wu and H.Z. Tang, Physical-constraint-preserving central discontinuous Galerkin methods for special relativistic hydrodynamics with a general equation of state, Astrophys. J. Suppl. Ser., 228 (2016), 3 .

[63] K.L. Wu and H.Z. Tang, Admissible state and physical-constraints-preserving schemes for relativistic magnetohydrodynamic equations, Math. Models Methods Appl. Sci., 27 (2017), $1871-1928$.

[64] K.L. Wu and H.Z. Tang, On physical-constraints-preserving schemes for special relativistic magnetohydrodynamics with a general equation of state, Z. Angew. Math. Phys., 69 (2018), $1-24$.

[65] K.L. Wu, Z.C. Yang, and H.Z. Tang, A third-order accurate direct Eulerian GRP scheme for one-dimensional relativistic hydrodynamics, East Asian J. Appl. Math., 4 (2014), 95-131. 
[66] X.B. Yang, W.Z. Huang, and J.X. Qiu, A moving mesh WENO method for one-dimensional conservation laws, SIAM J. Sci. Comput., 34 (2012), A2317-A2343.

[67] Z.C. Yang, P. He, and H.Z. Tang, A direct Eulerian GRP scheme for relativistic hydrodynamics: One-dimensional case, J. Comput. Phys., 230 (2011), 7964-7987.

[68] Z.C. Yang and H.Z. Tang, A direct Eulerian GRP scheme for relativistic hydrodynamics: Two-dimensional case, J. Comput. Phys., 231 (2012), 2116-2139.

[69] Y.H. Yuan and H.Z. Tang, Two-stage fourth-order accurate time discretizations for 1D and 2D special relativistic hydrodynamics, J. Comput. Math., 38 (2020), 768-796.

[70] H. Zhang, M. Reggio, J.Y. Trpanier, and R. Camarero, Discrete form of the GCL for moving meshes and its implementation in CFD schemes, Comput. \& Fluids, 22 (1993), 9-23.

[71] M. Zhang, J. Cheng, W.Z. Huang, and J.X. Qiu, An adaptive moving mesh discontinuous Galerkin method for the radiative transfer equation, Commun. Comput. Phys., 27 (2020), 1140-1173.

[72] W.Q. Zhang and A.I. MacFadyen, RAM: A relativistic adaptive mesh refinement hydrodynamics code, Astrophys. J. Suppl. Ser., 164 (2006), 255-279.

[73] J. Zhao and H.Z. Tang, Runge-Kutta discontinuous Galerkin methods with WENO limiter for the special relativistic hydrodynamics, J. Comput. Phys., 242 (2013), 138-168. 